\title{
Finite irreducible conformal modules over the Lie conformal superalgebra $\mathcal{S}(p) \rrbracket$
}

\author{
Haibo Chen*, Yanyong Hong and Yucai Su
}

\begin{abstract}
In the present paper, we introduce a class of infinite Lie conformal superalgebras $\mathcal{S}(p)$, which are closely related to Lie conformal algebras of extended Block type defined in [6. Then all finite nontrivial irreducible conformal modules over $\mathcal{S}(p)$ for $p \in \mathbb{C}^{*}$ are completely classified. As an application, we also present the classifications of finite non-trivial irreducible conformal modules over finite quotient algebras $\mathfrak{s}(n)$ for $n \geq 1$ and $\mathfrak{s h}$ which is isomorphic to a subalgebra of Lie conformal algebra of $N=2$ superconformal algebra. Moreover, as a generalized version of $\mathcal{S}(p)$, the infinite Lie conformal superalgebras $\mathcal{G S}(p)$ are constructed, which have a subalgebra isomorphic to the finite Lie conformal algebra of $N=2$ superconformal algebra.
\end{abstract}

Key words: Lie conformal superalgebra, finite conformal module, irreducible

Mathematics Subject Classification (2010): 17B10, 17B65, $17 \mathrm{~B} 68$.

\section{Contents}

1 Introduction

2 Preliminaries 可

3 Lie conformal superalgebra $\mathcal{S}(p)$ 卢

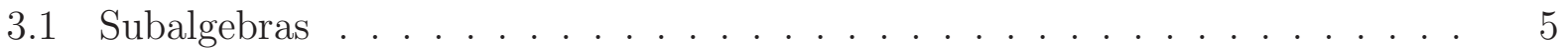

3.2 Quotient algebras ....................... . . . . . . . . . . .

3.3 Representation of annihilation superalgebras . . . . . . . . . . . 苗

4 Classification of finite irreducible modules 10

4.1 Equivalence of modules . . . . . . . . . . . . . . . . . 10

4.2 Rank $1+1$ modules . . . . . . . . . . . . . . . . . . . . . . 14

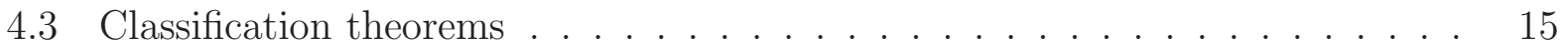

5 Generalized version of $\mathcal{S}(p)$

6 Applications $\quad 20$

\section{Introduction}

The notion of Lie conformal (super)algebras was originally introduced by Kac in [16, 17], which encodes an axiomatic description of the operator product expansion (or rather its Fourier transform) of chiral fields in conformal field theory. It plays important roles in quantum field theory, vertex algebras, integrable systems and so on, and has drawn many researchers' extensive attentions. As is well known, the theory of Lie conformal (super)algebras

${ }^{*}$ Corresponding author: Haibo Chen (rebel1025@126.com). 
gives us a powerful tool for the study of infinite-dimensional Lie (super)algebras satisfying the locality property described in [18].

There are a lot of researches on the case of Lie conformal algebra. It follows from [11] that Virasoro Lie conformal algebra and all current Lie conformal algebras with finite-dimensional simple Lie algebras exhaust all finite simple Lie conformal algebras. The theory of finite Lie conformal algebras associated with Virasoro Lie conformal algebra were intensively studied (see, e.g., 1, 7, 8, 11, 19, 25]). Furthermore, the problem of classifying finite simple Lie conformal superalgebras was completely solved in [12]. It shows that any finite simple Lie conformal superalgebra is isomorphic to one of the Lie conformal superalgebras of the list, which includes current Lie conformal superalgebras over finite-dimensional simple Lie superalgebras, four series of "Virasoro-like" Lie conformal superalgebras and exceptional Lie conformal superalgebra $C K_{6}$. It is worth noting that finite non-trivial irreducible conformal modules of them were completely classified in [2, 4, 7, 9, 21].

The infinite Lie conformal (super)algebra has become one of the major research objects in conformal algebraic theory. In order to better understand the theory of infinite Lie conformal (super)algebras, it is very natural to investigate some important examples. Based on the relation of Lie algebras and Lie conformal algebras, some infinite Lie conformal algebras were defined by loop Lie algebras and Block type Lie algebras (see, e.g., [5, 6, 14, 15, 22, 24]). In a similar way, a class of infinite Lie conformal superalgebras called loop Virasoro conformal superalgebras were constructed in [10, which are associated with the loop super-Virasoro algebras. Recently, the infinite Lie conformal superalgebras of Block type were introduced in [26], which contain a Neveu-Schwarz conformal subalgebra. At the same times, their finite non-trivial irreducible conformal modules were classified for $p \neq 0$. But, for all we know, there are very few works about the infinite Lie conformal superalgebras.

In this paper, we define a new class of infinite Lie conformal superalgebras $\mathcal{S}(p)$ with $p \neq$ 0 , which is related to a class of extended Block type Lie conformal algebras $\mathfrak{B}(\alpha, \beta, p)$ studied in [6]. The Lie conformal superalgebras of extended Block type are $\mathcal{S}(p)=\mathcal{S}(p)_{\overline{0}} \oplus \mathcal{S}(p)_{\overline{1}}$ with $\mathcal{S}(p)_{\overline{0}}=\oplus_{i \in \mathbb{Z}_{+}} \mathbb{C}[\partial] L_{i} \bigoplus \oplus_{i \in \mathbb{Z}_{+}} \mathbb{C}[\partial] W_{i}, \mathcal{S}(p)_{\overline{1}}=\oplus_{i \in \mathbb{Z}_{+}} \mathbb{C}[\partial] G_{i}$ and $\lambda$-brackets as follows

$$
\begin{aligned}
& {\left[L_{i \lambda} L_{j}\right]=((i+p) \partial+(i+j+2 p) \lambda) L_{i+j},} \\
& {\left[L_{i \lambda} W_{j}\right]=((i+p) \partial+(i+j+p) \lambda) W_{i+j},} \\
& {\left[L_{i \lambda} G_{j}\right]=((i+p) \partial+(i+j+2 p) \lambda) G_{i+j},} \\
& {\left[W_{i \lambda} G_{j}\right]=G_{i+j}, \quad\left[W_{i \lambda} W_{j}\right]=\left[G_{i \lambda} G_{j}\right]=0}
\end{aligned}
$$

for $i, j \in \mathbb{Z}_{+}$. Note that the even part $\mathcal{S}(p)_{\overline{0}}$ of $\mathcal{S}(p)$ is an extended Block type Lie conformal algebra $\mathfrak{B}(\alpha, \beta, p)$ for $\alpha=p, \beta=0$. The subalgebra $\mathfrak{H}=\mathbb{C}[\partial]\left(\frac{1}{p} L_{0}\right) \oplus \mathbb{C}[\partial] W_{0}$ of $\mathcal{S}(p)_{\overline{0}}$ is so-called Heisenberg-Virasoro Lie conformal algebra.

This article is organized as follows. 
In Section 2, we introduce some basic definitions and related known results about Lie conformal superalgebras and conformal modules.

In Section 3, by recalling the definition of $\mathfrak{B}(\alpha, \beta, p)$ and certain module structures, we define a class of Lie conformal superalgebras $\mathcal{S}(p)$. Then we investigate their subalgebras, quotient algebras and representations of annihilation superalgebras.

In Section 4, the irreducibility of all free non-trivial rank $1+1$ modules over $\mathcal{S}(p)$ are determined. A complete classification of all finite non-trivial irreducible conformal modules of $\mathcal{S}(p)$ are given, which shows that they must be free of rank 1 or $1+1$.

In Section 5, we construct a class of Lie conformal superalgebras, which are generalizations of Lie conformal superalgebras $\mathcal{S}(p)$. They have some subalgebras, one of them is exactly the Lie conformal algebra of $N=2$ superconformal algebra.

At last, as a byproduct of our main result, we also obtain the classification of all finite non-trivial irreducible conformal modules over the subalgebra $\mathfrak{s h}$ and quotient algebras $\mathfrak{s}(n)$ for $n \geq 1$.

Throughout this paper, all vector spaces, linear maps and tensor products are assumed to be over complex field $\mathbb{C}$. We denote by $\mathbb{C}^{*}, \mathbb{Z}$ and $\mathbb{Z}_{+}$the sets of nonzero complex numbers, integers and nonnegative integers, respectively. Moreover, if $A$ is a vector space, the space of polynomials of $\lambda$ with coefficients in $A$ is denoted by $A[\lambda]$.

\section{Preliminaries}

In this section, we recall some basic concepts and results related to Lie conformal superalgebras and conformal modules in [11,16, 17].

We denote $\mathbb{Z}_{2}=\{\overline{0}, \overline{1}\}$. A vector space $U$ is called $\mathbb{Z}_{2}$-graded if $U=U_{\overline{0}} \oplus U_{\overline{1}}$, and $u \in U_{\bar{i}}$ is called $\mathbb{Z}_{2}$-homogenous and write $|u|=\bar{i}$.

Definition 2.1. A Lie conformal superalgebra $S=S_{\overline{0}} \oplus S_{\overline{1}}$ is a $\mathbb{Z}_{2}$-graded $\mathbb{C}[\partial]$-module endowed with a $\lambda$-bracket $\left[a_{\lambda} b\right]$ which defines a linear map $S_{\alpha} \otimes S_{\beta} \rightarrow \mathbb{C}[\lambda] \otimes S_{\alpha+\beta}$, where $\lambda$ is an indeterminate, and satisfy the following axioms:

$$
\begin{aligned}
& {\left[\partial a_{\lambda} b\right]=-\lambda\left[a_{\lambda} b\right], \quad\left[a_{\lambda} \partial b\right]=(\partial+\lambda)\left[a_{\lambda} b\right],} \\
& {\left[a_{\lambda} b\right]=-(-1)^{|a||b|}\left[b_{-\lambda-\partial} a\right],} \\
& {\left[a_{\lambda}\left[b_{\mu} c\right]\right]=\left[\left[a_{\lambda} b\right]_{\lambda+\mu} c\right]+(-1)^{|a||b|}\left[b_{\mu}\left[a_{\lambda} c\right]\right]}
\end{aligned}
$$

for all $c \in S, \mathbb{Z}_{2}$-homogenous elements $a, b$ in $S$, and $\alpha, \beta \in \mathbb{Z}_{2}$.

A Lie conformal superalgebra is called finite if it is finitely generated as a $\mathbb{C}[\partial]$-module, or else it is called infinite. 
Definition 2.2. A conformal module $M=M_{\overline{0}} \oplus M_{\overline{1}}$ over a Lie conformal superalgebra $S$ is $a \mathbb{Z}_{2}$-graded $\mathbb{C}[\partial]$-module endowed with a $\lambda$-action $S_{\alpha} \otimes M_{\beta} \rightarrow \mathbb{C}[\lambda] \otimes M_{\alpha+\beta}$ such that

$$
\begin{aligned}
& (\partial a)_{\lambda} v=-\lambda a_{\lambda} v, a_{\lambda}(\partial v)=(\partial+\lambda) a_{\lambda} v, \\
& a_{\lambda}\left(b_{\mu} v\right)-(-1)^{|a||b|} b_{\mu}\left(a_{\lambda} v\right)=\left[a_{\lambda} b\right]_{\lambda+\mu} v
\end{aligned}
$$

for all $v \in M, \mathbb{Z}_{2}$-homogenous elements $a, b$ in $S$, and $\alpha, \beta \in \mathbb{Z}_{2}$.

Let $M=M_{\overline{0}} \oplus M_{\overline{1}}$ be a conformal $S$-module. Obviously, there is a parity-change functor $\Pi$ from the category of $S$-modules to itself, which implies that a new module $\Pi(M)$ is obtained by $\Pi\left(M_{\overline{0}}\right)=M_{\overline{1}}$ and $\Pi\left(M_{\overline{1}}\right)=M_{\overline{0}}$. The module $M$ is called finite if it is finitely generated over $\mathbb{C}[\partial]$. We call that the rank of $M$ is $m+n$ as a $\mathbb{C}[\partial]$-module, if the rank of $M_{\overline{0}}$ is $m$ and the rank of $M_{\overline{1}}$ is $n$. If $M$ has no non-trivial submodules, the conformal module $M$ is called irreducible.

A Lie conformal superalgebra $S$ is called $\mathbb{Z}$-graded if $S=\oplus_{i \in \mathbb{Z}} S_{i}$, each $S_{i}$ is a $\mathbb{C}[\partial]$ submodule and $\left[S_{i \lambda} S_{j}\right] \subseteq S_{i+j}[\lambda]$ for any $i, j \in \mathbb{Z}$. The conformal module $M$ is $\mathbb{Z}$-graded if $M=\oplus_{i \in \mathbb{Z}} M_{i}$, each $M_{i}$ is a $\mathbb{C}[\partial]$-submodule and $S_{i \lambda} M_{j} \subseteq M_{i+j}[\lambda]$ for any $i, j \in \mathbb{Z}$. Furthermore, if each $M_{i}$ is freely generated by an element $v_{i} \in M_{i}$ over $\mathbb{C}[\partial]$, then $M$ is called a $\mathbb{Z}$-graded free intermediate series module.

Definition 2.3. An annihilation superalgebra $\mathcal{A}(S)$ of a Lie conformal superalgebra $S$ is a Lie superalgebra with $\mathbb{C}$-basis $\left\{a(n) \mid a \in S, n \in \mathbb{Z}_{+}\right\}$and relations (for any $a, b \in S$ and $k \in \mathbb{C})$

$$
\begin{aligned}
& (k a)_{(n)}=k a_{(n)}, \quad(a+b)_{(n)}=a_{(n)}+b_{(n)}, \\
& {\left[a_{(m)}, b_{(n)}\right]=\sum_{k \in \mathbb{Z}_{+}}\left(\begin{array}{c}
m \\
k
\end{array}\right)\left(a_{(k)} b\right)_{(m+n-k)},(\partial a)_{(n)}=-n a_{(n-1)},}
\end{aligned}
$$

where $a(n) \in \mathcal{A}(S)_{\alpha}$ if $a \in S_{\alpha}$, and $a_{(k)} b$ is called the $k$-th product, given by $\left[a_{\lambda} b\right]=$ $\sum_{k \in \mathbb{Z}_{+}} \frac{\lambda^{k}}{k !}\left(a_{(k)} b\right)$. Furthermore, an extended annihilation superalgebra $\mathcal{A}(S)^{e}$ of $S$ is defined by $\mathcal{A}(S)^{e}=\mathbb{C} \partial \ltimes \mathcal{A}(S)$ with $\left[\partial, a_{(n)}\right]=-n a_{n-1}$, where $\mathbb{C} \partial \subseteq \mathcal{A}(S) \frac{e}{0}$.

Now we can define $k$-th actions of $S$ on $M$ for each $j \in \mathbb{Z}_{+}$, i.e. $a_{(k)} v$ for any $a \in S, v \in M$

$$
a_{\lambda} v=\sum_{k \in \mathbb{Z}_{+}} \frac{\lambda^{(k)}}{k !}\left(a_{(k)} v\right),
$$

which is similar to the definition of $k$-th product $a_{(k)} b$ for $a, b \in S$.

The following result appeared in [7, which implies that a close connection between the module of a Lie conformal superalgebra and that of its extended annihilation superalgebra.

Proposition 2.4. A conformal module $M$ over a Lie conformal superalgebra $S$ is the same as a module over the Lie superalgebra $\mathcal{A}(S)^{e}$ satisfying $a_{(n)} v=0$ for $a \in S, v \in M, n \gg 0$. 


\section{$3 \quad$ Lie conformal superalgebra $\mathcal{S}(p)$}

In this section, a class of extended Block type Lie conformal superalgebras $\mathcal{S}(p)$ are defined.

We first recall the definition of the extended Block type Lie conformal algebra $\mathfrak{B}(\alpha, \beta, p)$ and its $\mathbb{Z}$-graded intermediate series modules (see [6, 26]).

The infinite Lie conformal algebra called extended Block type Lie conformal algebra $\mathfrak{B}(\alpha, \beta, p)$ with $p \in \mathbb{C}^{*}$ has a $\mathbb{C}[\partial]$-basis $\left\{L_{i}, W_{i} \mid i \in \mathbb{Z}_{+}\right\}$satisfying the following nontrivial $\lambda$-brackets

$$
\begin{aligned}
& {\left[L_{i \lambda} L_{j}\right]=((i+p) \partial+(i+j+2 p) \lambda) L_{i+j},} \\
& {\left[L_{i \lambda} W_{j}\right]=((i+p)(\partial+\beta)+(i+j+\alpha) \lambda) W_{i+j}}
\end{aligned}
$$

for any $\alpha, \beta \in \mathbb{C}$. In the following, we only consider $\alpha=p, \beta=0$. For $\alpha_{1}, \beta_{1}, \gamma_{1} \in \mathbb{C}, p \in \mathbb{C}^{*}$, the $\mathbb{C}[\partial]$-module $V\left(\alpha_{1}, \beta_{1}, \gamma_{1}, p\right)=\bigoplus_{i \in \mathbb{Z}} \mathbb{C}[\partial] v_{i}$ is a $\mathbb{Z}$-graded free intermediate series module over $\mathfrak{B}(\alpha, \beta, p)$ with $\lambda$-actions as follows:

$$
L_{i \lambda} v_{j}=\left((i+p)\left(\partial+\beta_{1}\right)+\left(i+j+\alpha_{1}\right) \lambda\right) v_{i+j}, W_{i \lambda} v_{j}=\gamma_{1} v_{i+j}
$$

Inspired by this, we consider a $\mathbb{Z}_{2}$-graded $\mathbb{C}[\partial]$-module

$$
\mathcal{S}\left(\alpha_{1}, \beta_{1}, \gamma_{1}, p\right)=\mathcal{S}_{\overline{0}} \oplus \mathcal{S}_{\overline{1}}
$$

with $\mathcal{S}_{\overline{0}}=\oplus_{i \in \mathbb{Z}_{+}} \mathbb{C}[\partial] L_{i} \bigoplus \oplus_{i \in \mathbb{Z}_{+}} \mathbb{C}[\partial] W_{i}, \mathcal{S}_{\overline{1}}=\oplus_{i \in \mathbb{Z}_{+}} \mathbb{C}[\partial] G_{i}$, and satisfying

$$
\begin{aligned}
& {\left[L_{i \lambda} G_{j}\right]=\left((i+p)\left(\partial+\beta_{1}\right)+\left(i+j+\alpha_{1}\right) \lambda\right) G_{i+j},} \\
& {\left[W_{i \lambda} G_{j}\right]=\gamma_{1} G_{i+j},\left[G_{i \lambda} G_{j}\right]=0}
\end{aligned}
$$

for $i, j \in \mathbb{Z}_{+}$. Let $\alpha_{1}=2 p, \beta_{1}=0$ and $\gamma_{1}=1$. Then the $\mathbb{Z}_{2}$-graded $\mathbb{C}[\partial]$-module $\mathcal{S}\left(\alpha_{1}, \beta_{1}, \gamma_{1}, p\right)$ becomes the Lie conformal superalgebra $\mathcal{S}(p)$, which is exactly what we defined in (1.1)-(1.4).

Now we present some interesting features on $\mathcal{S}(p)$ as follows.

\subsection{Subalgebras}

Setting $L=\frac{1}{p} L_{0}, W=W_{0}, G=G_{0} \in \mathcal{S}(p)$ in (1.1)-(1.4), we can obtain the non-vanishing relations as follows:

$$
\left[L_{\lambda} L\right]=(\partial+2 \lambda) L,\left[L_{\lambda} W\right]=(\partial+\lambda) W,\left[L_{\lambda} G\right]=(\partial+2 \lambda) G,\left[W_{\lambda} G\right]=G
$$

which is called Heisenberg-Virasoro Lie conformal superalgebra $\mathfrak{s h}$. Clearly, the even part of $\mathfrak{s h}$ is Heisenberg-Virasoro Lie conformal algebra, which has been studied extensively (see, 
e.g., [6, 19, 25]). We see that $\mathbb{C}[\partial](L+a W)$ for $a \in \mathbb{C}$ spans a subalgebra of HeisenbergVirasoro Lie conformal algebra which is isomorphic to the Virasoro Lie conformal algebra. Now we define the following $\mathbb{C}[\partial]$-module homomorphism from $\mathfrak{s h}$ to Lie conformal algebra of $N=2$ superconformal algebra (see [9]):

$$
L+\frac{1}{2} \partial W \rightarrow L, W \rightarrow J, G \rightarrow G^{+}
$$

Then it is easy to check that $\mathfrak{s h}$ is isomorphic to a subalgebra of Lie conformal algebra of $N=2$ superconformal algebra.

\subsection{Quotient algebras}

Many finite Lie conformal superalgebras will be obtained by considering the quotient algebras of $\mathcal{S}(p)$. We note that $\mathcal{S}(p)$ is $\mathbb{Z}$-graded in the sense of

$$
\mathcal{S}(p)=\oplus_{k \in \mathbb{Z}_{+}} \mathcal{S}(p)_{k}
$$

where $\mathcal{S}(p)_{k}=\mathbb{C}[\partial] L_{k} \oplus \mathbb{C}[\partial] W_{k} \oplus \mathbb{C}[\partial] G_{k}$. For any $n \in \mathbb{Z}_{+}$, we can define a subspace $\mathcal{S}(p)_{\langle n\rangle}$ of $\mathcal{S}(p)$ by

$$
\mathcal{S}(p)_{\langle n\rangle}=\oplus_{i \geq n} \mathbb{C}[\partial] L_{i} \bigoplus \oplus_{i \geq n} \mathbb{C}[\partial] W_{i} \bigoplus \oplus_{i \geq n} \mathbb{C}[\partial] G_{i}
$$

Obviously, $\mathcal{S}(p)_{\langle n\rangle}$ is an ideal of the Lie conformal superalgebra of $\mathcal{S}(p)$. For $n \in \mathbb{Z}_{+}$, define

$$
\mathcal{S}(p)_{[n]}=\mathcal{S}(p) / \mathcal{S}(p)_{\langle n+1\rangle}
$$

Note that $\mathcal{S}(p)_{[0]} \cong \mathfrak{s h}$. Choosing $p=-n$ for $1 \leq n \in \mathbb{Z}$, we can define the quotient algebras $\mathcal{S}(-n)_{[n]}$ by the following relations

$$
\mathfrak{s}(n)=\mathcal{S}(-n)_{[n]}=\mathcal{S}(-n) / \mathcal{S}(-n)_{\langle n+1\rangle} .
$$

Then a series of new finite non-simple Lie conformal superalgebras can be produced. Now we give the following two examples for $n=1,2$.

Example 3.1. Setting $L=-\bar{L}_{0}, W=\bar{W}_{0}, G=\bar{G}_{0}, M=\bar{L}_{1}, H=\bar{W}_{1}, I=\bar{G}_{1} \in \mathfrak{s}(1)$, one can obtain the following non-trivial relations

$$
\begin{aligned}
& {\left[L_{\lambda} L\right]=(\partial+2 \lambda) L,\left[L_{\lambda} W\right]=(\partial+\lambda) W,\left[L_{\lambda} G\right]=(\partial+2 \lambda) G,} \\
& {\left[W_{\lambda} G\right]=G,\left[L_{\lambda} M\right]=(\partial+\lambda) M,\left[L_{\lambda} H\right]=\partial H} \\
& {\left[L_{\lambda} I\right]=(\partial+\lambda) I,\left[W_{\lambda} I\right]=\left[H_{\lambda} G\right]=I,\left[M_{\lambda} G\right]=-\lambda I .}
\end{aligned}
$$

Other $\lambda$-brackets are given by skew-symmetry. We observe that $\mathbb{C}[\partial] L \oplus \mathbb{C}[\partial] W$ and $\mathbb{C}[\partial] L \oplus$ $\mathbb{C}[\partial] M$ are both Heisenberg-Virasoro Lie conformal algebra. Maybe $\mathfrak{s}(1)$ should be called BiHeisenberg-Virasoro Lie conformal superalgebra. 
Example 3.2. Set $L=-\frac{1}{2} \bar{L}_{0}, W=\bar{W}_{0}, G=\bar{G}_{0}, M=\bar{L}_{1}, H=\bar{W}_{1}, I=\bar{G}_{1}, X=-\bar{L}_{2}, Y=$ $-\bar{W}_{2}, Z=-\bar{G}_{2} \in \mathfrak{s}(2)$. Then the non-vanishing relations are presented as follows

$$
\begin{aligned}
& {\left[L_{\lambda} L\right]=(\partial+2 \lambda) L,\left[L_{\lambda} W\right]=(\partial+\lambda) W,\left[L_{\lambda} G\right]=(\partial+2 \lambda) G,} \\
& {\left[W_{\lambda} G\right]=G,\left[L_{\lambda} M\right]=\left(\partial+\frac{3}{2} \lambda\right) M,\left[L_{\lambda} H\right]=\left(\partial+\frac{1}{2} \lambda\right) H,} \\
& {\left[L_{\lambda} I\right]=\left(\partial+\frac{3}{2} \lambda\right) I,\left[W_{\lambda} I\right]=I,\left[L_{\lambda} X\right]=(\partial+\lambda) X,\left[L_{\lambda} Y\right]=\partial Y,} \\
& {\left[L_{\lambda} Z\right]=(\partial+\lambda) Z,\left[W_{\lambda} Z\right]=Z,\left[M_{\lambda} M\right]=(\partial+2 \lambda) X,\left[M_{\lambda} H\right]=\partial Y,} \\
& {\left[M_{\lambda} I\right]=(\partial+2 \lambda) Z,\left[H_{\lambda} I\right]=Z,\left[M_{\lambda} W\right]=-(\partial+\lambda) H,} \\
& {\left[M_{\lambda} G\right]=-(\partial+3 \lambda) I,\left[H_{\lambda} G\right]=I,\left[X_{\lambda} G\right]=-2 \lambda Z,\left[Y{ }_{\lambda} G\right]=Z .}
\end{aligned}
$$

Other $\lambda$-brackets can be obtained by skew-symmetry. Note that $\mathbb{C}[\partial] L \oplus \mathbb{C}[\partial] M \oplus \mathbb{C}[\partial] X$ and $\mathbb{C}[\partial] L \oplus \mathbb{C}[\partial] W$ are respectively Schrödinger-Virasoro Lie conformal algebra and HeisenbergVirasoro Lie conformal algebra. Maybe $\mathfrak{s}(2)$ should be called Schrödinger-Heisenberg-Virasoro Lie conformal superalgebra.

\subsection{Representation of annihilation superalgebras}

In this section, the irreducible modules over a subquotient algebra of the annihilation superalgebra $\mathcal{A}(\mathcal{S}(p))$ of $\mathcal{S}(p)$ are classified.

Firstly, we provide the explicit super-brackets of $\mathcal{A}(\mathcal{S}(p))$ as follows.

Lemma 3.3. (1) The annihilation superalgebra of $\mathcal{S}(p)$ is

$$
\mathcal{A}(\mathcal{S}(p))=\left\{L_{i, m}, W_{j, n}, G_{k, l} \mid i, j, k, n \in \mathbb{Z}_{+}, m, l \in \mathbb{Z}_{+} \cup\{-1\}\right\}
$$

with the following Lie super-brackets:

$$
\begin{aligned}
& {\left[L_{i, m}, L_{j, n}\right]=((m+1)(j+p)-(n+1)(i+p)) L_{i+j, m+n},} \\
& {\left[L_{i, m}, W_{j, n}\right]=((m+1) j-n(i+p)) W_{i+j, m+n},} \\
& {\left[L_{i, m}, G_{j, n}\right]=((m+1)(j+p)-(n+1)(i+p)) G_{i+j, m+n},} \\
& {\left[W_{i, m}, G_{j, n}\right]=G_{i+j, m+n}, \quad\left[W_{i, m}, W_{j, n}\right]=\left[G_{i, m}, G_{j, n}\right]=0,}
\end{aligned}
$$

where $p \in \mathbb{C}^{*}$;

(2) The extended annihilation algebra is

$$
\mathcal{A}(\mathcal{S}(p))^{e}=\left\{L_{i, m}, W_{j, n}, G_{k, l}, \partial \mid i, j, k, n \in \mathbb{Z}_{+}, m, l \in \mathbb{Z}_{+} \cup\{-1\}\right\}
$$

satisfying (3.6) and

$$
\left[\partial, L_{i, m}\right]=-(m+1) L_{i, m-1},\left[\partial, W_{j, n}\right]=-n W_{j, n-1}, \quad\left[\partial, G_{k, l}\right]=-(l+1) G_{k, l-1} .
$$


Proof. By the definition of the $k$-th product in Definition 2.3 and $\mathcal{S}(p)$, we conclude that

$$
\begin{aligned}
& L_{i(k)} L_{j}= \begin{cases}(i+p) \partial L_{i+j} & \text { if } k=0 \\
(i+j+2 p) L_{i+j} & \text { if } k=1 \\
0 & \text { if } k \geq 2\end{cases} \\
& L_{i(k)} W_{j}= \begin{cases}(i+p) \partial W_{i+j} & \text { if } k=0, \\
(i+j+p) W_{i+j} & \text { if } k=1, \\
0 & \text { if } k \geq 2,\end{cases} \\
& L_{i(k)} G_{j}= \begin{cases}(i+p) \partial G_{i+j} & \text { if } k=0, \\
(i+j+2 p) G_{i+j} & \text { if } k=1, \\
0 & \text { if } k \geq 2,\end{cases} \\
& W_{i(k)} G_{j}= \begin{cases}G_{i+j} & \text { if } k=0, \\
0 & \text { if } k \geq 1,\end{cases} \\
& G_{i_{(k)}} G_{j}=W_{i_{(k)}} W_{j}=0 \text { for any } k \in \mathbb{Z}_{+} \text {. }
\end{aligned}
$$

From (2.1) and (2.2), it is easy to see that

$$
\begin{aligned}
& {\left[\left(L_{i}\right)_{(m)},\left(L_{j}\right)_{(n)}\right]=(m(j+p)-n(i+p))\left(L_{i+j}\right)_{(m+n-1)},} \\
& {\left[\left(L_{i}\right)_{(m)},\left(W_{j}\right)_{(n)}\right]=(m j-n(i+p))\left(W_{i+j}\right)_{(m+n-1)},} \\
& {\left[\left(L_{i}\right)_{(m)},\left(G_{j}\right)_{(n)}\right]=(m(j+p)-n(i+p))\left(G_{i+j}\right)_{(m+n-1)},} \\
& {\left[\left(W_{i}\right)_{(m)},\left(G_{j}\right)_{(n)}\right]=\left(G_{i+j}\right)_{(m+n)},\left[\left(G_{i}\right)_{(m)},\left(G_{j}\right)_{(n)}\right]=0,} \\
& {\left[\left(W_{i}\right)_{(m)},\left(W_{j}\right)_{(n)}\right]=0,\left[\partial,\left(L_{i}\right)_{(m)}\right]=-m\left(L_{i}\right)_{(m-1)},} \\
& {\left[\partial,\left(W_{j}\right)_{(n)}\right]=-n\left(W_{j}\right)_{(n-1)},\left[\partial,\left(G_{k}\right)_{(l)}\right]=-l\left(G_{k}\right)_{(l-1)} .}
\end{aligned}
$$

Then the lemma is proved by setting $L_{i, m}=\left(L_{i}\right)_{(m+1)}, W_{j, n}=\left(W_{j}\right)_{(n)}$ and $G_{k, l}=\left(G_{k}\right)_{(l+1)}$ in (3.7) for $i, j, k, n \in \mathbb{Z}_{+}, m, l \in \mathbb{Z}_{+} \cup\{-1\}$.

Remark 3.4. It has come to our notice that the super Heisenberg-Virasoro algebra is isomorphic to the Lie superalgebra generated by $\left\{L_{0, m}, W_{0, n}, G_{0, l} \mid m, n, l \in \mathbb{Z}\right\}$ in $\mathcal{A}(\mathcal{S}(p))$ (see [20]).

Secondly, we investigate the representation theory of a subquotient algebra of $\mathcal{A}(\mathcal{S}(p))$. It is obvious that

$$
\mathcal{A}(\mathcal{S}(p))^{+}=\mathcal{A}(\mathcal{S}(p))_{\overline{0}}^{+} \oplus \mathcal{A}(\mathcal{S}(p))_{\overline{1}}^{+}
$$


is a subalgebra of $\mathcal{A}(\mathcal{S}(p))$, where $\mathcal{A}(\mathcal{S}(p))_{\overline{0}}^{+}=\left\{L_{i, m}, W_{j, n} \mid i, j, m, n \in \mathbb{Z}_{+}\right\}$and $\mathcal{A}(\mathcal{S}(p))_{\overline{1}}^{+}=$ $\left\{G_{k, l} \mid k, l \in \mathbb{Z}_{+}\right\}$. For any $t, N \in \mathbb{Z}_{+}$, we denote

$$
\mathcal{I}(t, N)=\mathcal{I}(k, N)_{\overline{0}} \oplus \mathcal{I}(k, N)_{\overline{1}}
$$

where $\mathcal{I}(t, N)_{\overline{0}}=\left\{L_{i, m}, W_{j, n} \in \mathcal{A}(\mathcal{S}(p))_{+} \mid i, j>t, m, n>N\right\}$ and $\mathcal{I}(t, N)_{\overline{1}}=\left\{G_{k, l} \in\right.$ $\left.\mathcal{A}(\mathcal{S}(p))_{+} \mid k>t, l>N\right\}$. We see that $\mathcal{I}(t, N)$ is an ideal of $\mathcal{A}(\mathcal{S}(p))^{+}$. Denote

$$
\mathfrak{p}(t, N)=\mathfrak{p}(t, N)_{\overline{0}} \oplus \mathfrak{p}(t, N)_{\overline{1}}=\mathcal{A}(\mathcal{S}(p))^{+} / \mathcal{I}(t, N)
$$

For the later use, denote the following ideals of $\mathfrak{p}(t, N)$ for $t, N \geq 1$ :

$$
\begin{aligned}
& \chi(t, N)=\operatorname{span}_{\mathbb{C}}\left\{\bar{L}_{t, m}, \bar{W}_{t, n}, \bar{G}_{t, l} \in \mathfrak{p}(t, N) \mid m, n, l \leq N\right\}, \\
& \psi(t, N)=\operatorname{span}_{\mathbb{C}}\left\{\bar{L}_{i, N}, \bar{W}_{j, N}, \bar{G}_{k, N} \in \mathfrak{p}(t, N) \mid i, j, k \leq t\right\}, \\
& \phi(t, N)=\operatorname{span}_{\mathbb{C}}\left\{\bar{L}_{t, m}, \bar{W}_{t, n}, \bar{G}_{t, l}, \bar{L}_{i, N}, \bar{W}_{j, N}, \bar{G}_{k, N} \in \mathfrak{p}(t, N) \mid m, n, l \leq N, i, j, k \leq t-1\right\} .
\end{aligned}
$$

We also denote two finite sets:

$$
\Phi=\left\{(i, m) \mid \bar{L}_{i, m}, \bar{W}_{i, m}, \bar{G}_{i, m} \in \mathfrak{p}(t, N)\right\} \backslash\{(0,0)\}, \Phi_{0}=\{(i, m) \in \Phi \mid i-p m=0\} .
$$

Lemma 3.5. Let $t, N \geq 1, \Phi_{0} \neq \emptyset$ and

$$
i_{0}=\max \left\{i \mid(i, m) \in \Phi_{0}\right\}, m_{0}=\max \left\{m \mid(i, m) \in \Phi_{0}\right\} .
$$

Assume that $V=V_{\overline{0}} \oplus V_{\overline{1}}$ is a non-trivial finite-dimensional irreducible module over $\mathfrak{p}(t, N)$.

(1) If $i_{0}<t$, then the ideal $\chi(t, N)$ of $\mathfrak{p}(t, N)$ acts trivially on $V$;

(2) If $m_{0}<N$, then the ideal $\psi(t, N)$ of $\mathfrak{p}(t, N)$ acts trivially on $V$;

(3) If $i_{0}=t, m_{0}=N$, then the ideal $\phi(t, N)$ of $\mathfrak{p}(t, N)$ acts trivially on $V$.

Proof. (1) Consider the action of $\bar{L}_{0,0}$ on $\chi(t, N)$ :

$$
\left[\bar{L}_{0,0}, \bar{L}_{i, m}\right]=(i-m p) \bar{L}_{i, m},\left[\bar{L}_{0,0}, \bar{W}_{i, m}\right]=(i-m p) \bar{W}_{i, m},\left[\bar{L}_{0,0}, \bar{G}_{i, m}\right]=(i-m p) \bar{G}_{i, m}
$$

where $0 \leq i \leq t, 0 \leq m \leq N$. It follows from $t>i_{0}$ that $(t-m p) \neq 0$. Obviously, $\chi(t, N)$ is a completely reducible $\mathbb{C} \bar{L}_{0,0}$-module with no trivial summand. By Lemma 1 of [7], we get that $\chi(t, N)$ acts trivially on $V$. Similarly, (2) can be obtained.

(3) Note that $p>0$. Assume that $\phi(t, N)$ acts non-trivially on $V$. According to the irreducibility of $V$, one can see that $V=\phi(t, N) V$. Choose a decomposition of $\phi(t, N)$ as follows

$$
\phi(t, N)=\operatorname{span}_{\mathbb{C}}\left\{\bar{L}_{i_{0}, m_{0}}, \bar{W}_{i_{0}, m_{0}}, \bar{G}_{i_{0}, m_{0}}\right\}+\widetilde{\phi}(t, N),
$$

where $\widetilde{\phi}(t, N)=\phi(t, N) \backslash \operatorname{span}_{\mathbb{C}}\left\{\bar{L}_{i_{0}, m_{0}}, \bar{W}_{i_{0}, m_{0}}, \bar{G}_{i_{0}, m_{0}}\right\}$. 
Considering the action of $\bar{L}_{0,0}$ on $\widetilde{\phi}(t, N)$, we know that every element in $\widetilde{\phi}(t, N)$ acts nilpotently on $V$ by Lemma 1 of [7]. From

$$
\begin{aligned}
& {\left[\bar{L}_{i_{0}, 0}, \bar{L}_{0, m_{0}}\right]=-\left(\left(i_{0}+p\right) m_{0}+i_{0}\right) \bar{L}_{i_{0}, m_{0}},} \\
& {\left[\bar{L}_{i_{0}, 0}, \bar{W}_{0, m_{0}}\right]=-\left(i_{0}+p\right) m_{0} \bar{W}_{i_{0}, m_{0}},} \\
& {\left[\bar{L}_{i_{0}, 0}, \bar{G}_{0, m_{0}}\right]=-\left(\left(i_{0}+p\right) m_{0}+i_{0}\right) \bar{G}_{i_{0}, m_{0}},}
\end{aligned}
$$

we check that $\operatorname{span}_{\mathbb{C}}\left\{\bar{L}_{i_{0}, m_{0}}, \bar{W}_{i_{0}, m_{0}}, \bar{G}_{i_{0}, m_{0}}\right\}$ acts trivially on $V$. The results hold.

Lemma 3.6. Let $V=V_{\overline{0}} \oplus V_{\overline{1}}$ be a non-trivial finite-dimensional irreducible module over $\mathfrak{p}(t, N)$. Then we obtain $\operatorname{dim}(V)=\operatorname{dim}\left(V_{\overline{0}}\right)=1$ or $\operatorname{dim}(V)=1+1$.

Proof. Regard $V$ as a finite-dimensional $\mathfrak{p}(t, N) \overline{0^{-}}$-module. By Lemma 3.5 of [6], we know that there exists $v \in V$ such that $\bar{L}_{i, m} v=\sigma_{i, m} v, \bar{W}_{i, m} v=\tau_{i, m} v$ for all $i, m \in \mathbb{Z}_{+}$, where $\sigma_{i, m}, \tau_{i, m} \in \mathbb{C}$.

When $\bar{G}_{0,0} v=0$, by the relation of $\left[\bar{W}_{i, m}, \bar{G}_{0,0}\right]=\bar{G}_{i, m}$, we obtain $\bar{G}_{i, m} v=0$ for $i, m \in \mathbb{Z}_{+}$. Then $\operatorname{dim}(V)=\operatorname{dim}\left(V_{\overline{0}}\right)=1$.

If $\bar{G}_{0,0} v \neq 0$, we present the following two cases. First, consider $\Phi_{0}=\emptyset$. We have a decomposition of $\mathfrak{p}(t, N)$ :

$$
\mathfrak{p}(t, N)=\operatorname{span}_{\mathbb{C}}\left\{\bar{L}_{0,0}, \bar{W}_{0,0}, \bar{G}_{0,0}\right\}+\widetilde{\mathfrak{p}}(t, N),
$$

where $\widetilde{\mathfrak{p}}(t, N)=\mathfrak{p}(t, N) \backslash \operatorname{span}_{\mathbb{C}}\left\{\bar{L}_{0,0}, \bar{W}_{0,0}, \bar{G}_{0,0}\right\}$ and $\widetilde{\mathfrak{p}}(t, N)$ is a nilpotent ideal of $\mathfrak{p}(t, N)$. Considering the action of $\bar{L}_{0,0}$ on $\widetilde{\mathfrak{p}}(t, N)$, which implies that $\widetilde{\mathfrak{p}}(t, N)$ is a completely reducible $\mathbb{C} \bar{L}_{0,0}$-module with no trivial summand. By Lemma 1 of [7] and $\bar{G}_{0,0}^{2} v=0$, we immediately obtain that $V_{\overline{1}}=\mathbb{C} \bar{G}_{0,0} v$ and $\operatorname{dim}(V)=1+1$.

Next, consider $\Phi_{0} \neq \emptyset$. Assume that $t, N \geq 1$. We note that if the $\chi(t, N)$ (respectively, ideals $\psi(t, N), \phi(t, N))$ of $\mathfrak{p}(t, N)$ acts trivially on $V$, then $V$ can be viewed as an irreducible module over $\mathfrak{p}(t-1, N)$ (respectively, $\mathfrak{p}(t, N-1), \mathfrak{p}(t-1, N-1))$. Using simultaneous induction on $t, N$ and Lemma 3.5, we obtain that the odd vector can be written as $V_{\overline{1}}=$ $\mathbb{C} \bar{G}_{0,0} v$, and $\operatorname{dim}(V)=1+1$.

\section{Classification of finite irreducible modules}

This section will be devoted to giving a complete classification of all finite non-trivial irreducible conformal modules over $\mathcal{S}(p)$.

\subsection{Equivalence of modules}

We first recall a useful result appeared in [6], which is related to classification of finite non-trivial irreducible conformal modules over $\mathcal{S}(p)_{\overline{0}}$. 
Lemma 4.1. Assume that $V$ is a finite non-trivial irreducible conformal module over $\mathcal{S}(p)_{\overline{0}}$. Then $V$ is isomorphic to $V_{a, b, c, d}=\mathbb{C}[\partial] v$ with

$$
\left\{\begin{array}{l}
L_{0 \lambda} v=p(\partial+a \lambda+b) v, \\
L_{1 \lambda} v=\delta_{p+1,0} c v, \\
W_{0 \lambda} v=d v \\
W_{i \lambda} v=0, i \geq 1, \\
L_{j \lambda} v=0, j \geq 2
\end{array}\right.
$$

for $a, b, c, d \in \mathbb{C}$.

The equivalence between finite conformal modules over $\mathcal{S}(p)$ and those over its quotient algebra $\mathcal{S}(p)_{[n]}$ for some $n \in \mathbb{Z}_{+}$are given as follows.

Theorem 4.2. Let $V$ be a finite non-trivial conformal module over $\mathcal{S}(p)$. Then the $\lambda$-actions of $L_{i}, W_{i}$ and $G_{i}$ on $V$ are trivial for $i \gg 0$.

Proof. Regard $V$ as a finite conformal module over $\mathcal{S}(p)_{\overline{0}}$. It follows from Theorem 4.2 of [6] that $L_{i \lambda} v=W_{i \lambda} v=0$ for all $i \gg 0$ and any $v \in V$. Take $i$ such that $i>|p|$. Fix $i \gg 0$. Since

$$
W_{i \lambda}\left(G_{0 \mu} v\right)-G_{0 \mu}\left(W_{i \lambda} v\right)=G_{i \lambda+\mu} v,
$$

one can check that $G_{i \lambda} v=0$ for any $v \in V$, proving the theorem.

Remark 4.3. In fact, a finite conformal module over $\mathcal{S}(p)$ is isomorphic to a finite conformal module over $\mathcal{S}(p)_{[n]}$ for some large enough $n \in \mathbb{Z}$, where $\mathcal{S}(p)_{[n]}$ is defined as (3.4).

\subsection{Rank $1+1$ modules}

In the following, a characterization of non-trivial free conformal modules of rank $1+1$ over $\mathcal{S}(p)$ are presented. According to Lemma 4.1, we can define the following three classes of conformal modules $V_{a, b, c, d, a^{\prime}, b^{\prime}, c^{\prime}, d^{\prime}}, V_{a, b, c, d, \sigma}$ and $V_{a, b, \sigma}$.

(1) $V_{a, b, c, d, a^{\prime}, b^{\prime}, c^{\prime}, d^{\prime}}=\mathbb{C}[\partial] v_{\overline{0}} \oplus \mathbb{C}[\partial] v_{\overline{1}}$ with

$$
\left\{\begin{array} { l } 
{ L _ { 0 \lambda } v _ { \overline { 0 } } = p ( \partial + a \lambda + b ) v _ { \overline { 0 } } , } \\
{ L _ { 1 \lambda } v _ { \overline { 0 } } = c v _ { \overline { 0 } } , } \\
{ W _ { 0 \lambda } v _ { \overline { 0 } } = d v _ { \overline { 0 } } , } \\
{ G _ { i \lambda } v _ { \overline { 0 } } = 0 , i \geq 0 , } \\
{ W _ { j \lambda } v _ { \overline { 0 } } = 0 , j \geq 1 , } \\
{ L _ { k \lambda } v _ { \overline { 0 } } = 0 , k \geq 2 , }
\end{array} \quad \text { and } \left\{\begin{array}{l}
L_{0 \lambda} v_{\overline{1}}=p\left(\partial+a^{\prime} \lambda+b^{\prime}\right) v_{\overline{1}}, \\
L_{1 \lambda} v_{\overline{1}}=c^{\prime} v_{\overline{1}}, \\
W_{0 \lambda} v_{\overline{1}}=d^{\prime} v_{\overline{1}}, \\
G_{i \lambda} v_{\overline{1}}=0, i \geq 0, \\
W_{j \lambda} v_{\overline{1}}=0, j \geq 1, \\
L_{k \lambda} v_{\overline{1}}=0, k \geq 2,
\end{array}\right.\right.
$$


where $a, b, c, d, a^{\prime}, b^{\prime}, c^{\prime}, d^{\prime} \in \mathbb{C}$;

(2) $V_{a, b, c, d, \sigma}=\mathbb{C}[\partial] v_{\overline{0}} \oplus \mathbb{C}[\partial] v_{\overline{1}}$ with

$$
\left\{\begin{array} { l } 
{ L _ { 0 \lambda } v _ { \overline { 0 } } = p ( \partial + a \lambda + b ) v _ { \overline { 0 } } , } \\
{ L _ { 1 \lambda } v _ { \overline { 0 } } = c v _ { \overline { 0 } } , } \\
{ W _ { 0 \lambda } v _ { \overline { 0 } } = d v _ { \overline { 0 } } , } \\
{ G _ { 0 \lambda } v _ { \overline { 0 } } = \sigma v _ { \overline { 1 } } , } \\
{ G _ { i \lambda } v _ { \overline { 0 } } = 0 , i \geq 1 , } \\
{ W _ { j \lambda } v _ { \overline { 0 } } = 0 , j \geq 1 , } \\
{ L _ { k \lambda } v _ { \overline { 0 } } = 0 , k \geq 2 , }
\end{array} \quad \text { and } \left\{\begin{array}{l}
L_{0 \lambda} v_{\overline{1}}=p(\partial+(a+1) \lambda+b) v_{\overline{1}}, \\
L_{1 \lambda} v_{\overline{1}}=c v_{\overline{1}}, \\
W_{0 \lambda} v_{\overline{1}}=(d+1) v_{\overline{1}}, \\
G_{i \lambda} v_{\overline{1}}=0, i \geq 0, \\
W_{j \lambda} v_{\overline{1}}=0, j \geq 1, \\
L_{k \lambda} v_{\overline{1}}=0, k \geq 2,
\end{array}\right.\right.
$$

where $a, b, c, d \in \mathbb{C}, \sigma \in \mathbb{C}^{*}$;

(3) $V_{a, b, \sigma}=\mathbb{C}[\partial] v_{\overline{0}} \oplus \mathbb{C}[\partial] v_{\overline{1}}$ with

$$
\left\{\begin{array} { l } 
{ L _ { 0 \lambda } v _ { \overline { 0 } } = p ( \partial + a \lambda + b ) v _ { \overline { 0 } } , } \\
{ W _ { 0 \lambda } v _ { \overline { 0 } } = ( a - 1 ) v _ { \overline { 0 } } , } \\
{ G _ { 0 \lambda } v _ { \overline { 0 } } = \sigma ( \partial + a \lambda + b ) v _ { \overline { 1 } } , \quad \text { and } } \\
{ G _ { i \lambda } v _ { \overline { 0 } } = 0 , i \geq 1 , } \\
{ W _ { j \lambda } v _ { \overline { 0 } } = 0 , j \geq 1 , } \\
{ L _ { k \lambda } v _ { \overline { 0 } } = 0 , k \geq 1 , }
\end{array} \quad \left\{\begin{array}{l}
L_{0 \lambda} v_{\overline{1}}=p(\partial+a \lambda+b) v_{\overline{1}}, \\
W_{0 \lambda} v_{\overline{1}}=a v_{\overline{1}}, \\
G_{i \lambda} v_{\overline{1}}=0, i \geq 0, \\
W_{j \lambda} v_{\overline{1}}=0, j \geq 1, \\
L_{k \lambda} v_{\overline{1}}=0, k \geq 1,
\end{array}\right.\right.
$$

where $a, b \in \mathbb{C}, \sigma \in \mathbb{C}^{*}$.

Theorem 4.4. Let $V$ be a non-trivial free conformal module of rank $1+1$ over $\mathcal{S}(p)$.

(1) If $p \neq-1$, then $V \cong V_{a, b, 0, d, a^{\prime}, b^{\prime}, 0, d^{\prime}}$ or $V_{a, b, 0, d, \sigma}$ or $V_{a, b, \sigma}$ or $\Pi\left(V_{a-1, b, 0, d-1, \sigma}\right)$ or $\Pi\left(V_{a, b, \sigma}\right)$ for some $a, b, d, a^{\prime}, b^{\prime}, d^{\prime} \in \mathbb{C}, \sigma \in \mathbb{C}^{*}$;

(2) If $p=-1$, then $V \cong V_{a, b, c, d, a^{\prime}, b^{\prime}, c^{\prime}, d^{\prime}}$ or $V_{a, b, c, d, \sigma}$ or $V_{a, b, \sigma}$ or $\Pi\left(V_{a-1, b, c, d-1, \sigma}\right)$ or $\Pi\left(V_{a, b, \sigma}\right)$ for some $a, b, c, d, a^{\prime}, b^{\prime}, c^{\prime}, d^{\prime} \in \mathbb{C}, \sigma \in \mathbb{C}^{*}$.

Proof. In order to prove this theorem, we first let $V=\mathbb{C}[\partial] v_{\overline{0}} \oplus \mathbb{C}[\partial] v_{\overline{1}}$. Regarding $V$ as a conformal module over $\mathcal{S}(p)_{\overline{0}}$ and using Lemma 4.1, we can suppose that

$$
\left\{\begin{array} { l } 
{ L _ { 0 \lambda } v _ { \overline { 0 } } = p ( \partial + a \lambda + b ) v _ { \overline { 0 } } , } \\
{ L _ { 1 \lambda } v _ { \overline { 0 } } = \delta _ { p + 1 , 0 } c v _ { \overline { 0 } } , } \\
{ W _ { 0 \lambda } v _ { \overline { 0 } } = d v _ { \overline { 0 } } , } \\
{ W _ { i \lambda } v _ { \overline { 0 } } = 0 , i \geq 1 , } \\
{ L _ { j \lambda } v _ { \overline { 0 } } = 0 , j \geq 2 , }
\end{array} \quad \text { and } \left\{\begin{array}{l}
L_{0 \lambda} v_{\overline{1}}=p\left(\partial+a^{\prime} \lambda+b^{\prime}\right) v_{\overline{1}}, \\
L_{1 \lambda} v_{\overline{1}}=\delta_{p+1,0} c^{\prime} v_{\overline{1}}, \\
W_{0 \lambda} v_{\overline{1}}=d^{\prime} v_{\overline{1}} \\
W_{i \lambda} v_{\overline{1}}=0, i \geq 1, \\
L_{j \lambda} v_{\overline{1}}=0, j \geq 2
\end{array}\right.\right.
$$


where $a, b, c, d, a^{\prime}, b^{\prime}, c^{\prime}, d^{\prime} \in \mathbb{C}$. Owing to Theorem 4.2, we see that $L_{i \lambda} v_{s}=W_{i \lambda} v_{s}=$ $G_{i \lambda} v_{s}=0$ for $s \in \mathbb{Z}_{2}, i \gg 0$. Let $t_{0} \in \mathbb{Z}_{+}$be the largest integer such that the action of $\mathcal{S}(p)_{t_{0}}($ see $(\underline{3.3}))$ on $V$ is non-trivial. For $0 \leq t \leq t_{0}, t \in \mathbb{Z}_{+}$, we can write

$$
\begin{aligned}
& L_{t \lambda} v_{\overline{0}}=f_{t}(\partial, \lambda) v_{\overline{0}}, L_{t \lambda} v_{\overline{1}}=\widehat{f}_{t}(\partial, \lambda) v_{\overline{1}}, \\
& W_{t \lambda} v_{\overline{0}}=g_{t}(\partial, \lambda) v_{\overline{0}}, W_{t \lambda} v_{\overline{1}}=\widehat{g}_{t}(\partial, \lambda) v_{\overline{1}}, \\
& G_{t \lambda} v_{\overline{0}}=h_{t}(\partial, \lambda) v_{\overline{1}}, G_{t \lambda} v_{\overline{1}}=\widehat{h}_{t}(\partial, \lambda) v_{\overline{0}},
\end{aligned}
$$

where $f_{t}(\partial, \lambda), \widehat{f}_{t}(\partial, \lambda), g_{t}(\partial, \lambda), \widehat{g}_{t}(\partial, \lambda), h_{t}(\partial, \lambda), \widehat{h}_{t}(\partial, \lambda) \in \mathbb{C}[\partial, \lambda]$. Note that

$$
\begin{aligned}
& f_{0}(\partial, \lambda)=p(\partial+a \lambda+b), f_{1}(\partial, \lambda)=\delta_{p+1,0} c, f_{i}(\partial, \lambda)=0 \\
& \widehat{f}_{0}(\partial, \lambda)=p\left(\partial+a^{\prime} \lambda+b^{\prime}\right), \widehat{f}_{1}(\partial, \lambda)=\delta_{p+1,0} c^{\prime}, \widehat{f}_{i}(\partial, \lambda)=0 \\
& g_{0}(\partial, \lambda)=d, \widehat{g}_{0}(\partial, \lambda)=d^{\prime}, g_{j}(\partial, \lambda)=\widehat{g}_{j}(\partial, \lambda)=0
\end{aligned}
$$

for $i \geq 2, j \geq 1$. For $1 \leq t \leq t_{0}, s \in \mathbb{Z}_{2}$, we have

$$
W_{t \lambda}\left(G_{0 \mu} v_{s}\right)-G_{0 \mu}\left(W_{t \lambda} v_{s}\right)=G_{t \lambda+\mu} v_{s}
$$

which gives $h_{t}(\partial, \lambda)=\widehat{h}_{t}(\partial, \lambda)=0$ for $t \geq 1$. Then for $s \in \mathbb{Z}_{2}$, by $L_{1 \lambda}\left(G_{0 \mu} v_{s}\right)=$ $G_{0 \mu}\left(L_{1 \lambda} v_{s}\right)$, one has

$$
\left(h_{0}(\partial+\lambda, \mu) c^{\prime}-h_{0}(\partial, \mu) c\right) \delta_{p+1,0}=0 \quad \text { and } \quad\left(\widehat{h}_{0}(\partial+\lambda, \mu) c-\widehat{h}_{0}(\partial, \mu) c^{\prime}\right) \delta_{p+1,0}=0 .
$$

For $s \in \mathbb{Z}_{2}$, we have $G_{0 \lambda}\left(G_{0 \mu} v_{s}\right)+G_{0 \mu}\left(G_{0 \lambda} v_{s}\right)=0$, which implies

$$
\begin{aligned}
& h_{0}(\partial+\lambda, \mu) \widehat{h}_{0}(\partial, \lambda)+h_{0}(\partial+\mu, \lambda) \widehat{h}_{0}(\partial, \mu)=0, \\
& \widehat{h}_{0}(\partial+\lambda, \mu) h_{0}(\partial, \lambda)+\widehat{h}_{0}(\partial+\mu, \lambda) h_{0}(\partial, \mu)=0 .
\end{aligned}
$$

Setting $\lambda=\mu=0$ in (4.2) or (4.3), one can get $h_{0}(\partial, 0) \widehat{h}_{0}(\partial, 0)=0$. Let us consider the following three cases.

Case 1. $h_{0}(\partial, 0)=\widehat{h}_{0}(\partial, 0)=0$.

For $s \in \mathbb{Z}_{2}$, we get $L_{0 \lambda}\left(G_{0 \mu} v_{s}\right)-G_{0 \mu}\left(L_{0 \lambda} v_{s}\right)=\left[L_{0 \lambda} G_{0}\right]_{\lambda+\mu} v_{s}$, which shows

$$
\begin{aligned}
& h_{0}(\partial+\lambda, \mu)\left(\partial+a^{\prime} \lambda+b^{\prime}\right)-(\partial+\mu+a \lambda+b) h_{0}(\partial, \mu) \\
= & (\lambda-\mu) h_{0}(\partial, \lambda+\mu), \\
& \widehat{h}_{0}(\partial+\lambda, \mu)(\partial+a \lambda+b)-\left(\partial+\mu+a^{\prime} \lambda+b^{\prime}\right) \widehat{h}_{0}(\partial, \mu) \\
= & (\lambda-\mu) \widehat{h}_{0}(\partial, \lambda+\mu) .
\end{aligned}
$$

Setting $\mu=0$ in (4.4) and (4.5), we check that $h_{0}(\partial, \lambda)=\widehat{h}_{0}(\partial, \lambda)=0$. 
Case 2. $h_{0}(\partial, 0) \neq 0$ and $\widehat{h}_{0}(\partial, 0)=0$.

Taking $\mu=0$ in (4.5) gives $\widehat{h}_{0}(\partial, \lambda)=0$. Based on $W_{0 \lambda}\left(G_{0 \mu} v_{\overline{0}}\right)-G_{0 \mu}\left(W_{0 \lambda} v_{\overline{0}}\right)=$ $G_{0 \lambda+\mu} v_{\overline{0}}$, we obtain

$$
d^{\prime} h_{0}(\partial+\lambda, \mu)-d h_{0}(\partial, \mu)=h_{0}(\partial, \lambda+\mu)
$$

Setting $\lambda=\mu=0$ respectively in (4.4) and (4.6), we check that

$$
b^{\prime}=b \quad \text { and } \quad d^{\prime}=d+1 .
$$

Then we let $\mu=0$ in (4.4) and (4.6), which gives

$$
\begin{aligned}
& \left(\partial+a^{\prime} \lambda+b^{\prime}\right) h_{0}(\partial+\lambda, 0)-(\partial+a \lambda+b) h_{0}(\partial, 0)=\lambda h_{0}(\partial, \lambda) \\
& d^{\prime} h_{0}(\partial+\lambda, 0)-d h_{0}(\partial, 0)=h_{0}(\partial, \lambda) .
\end{aligned}
$$

Inserting (4.8) into (4.7), we get

$$
\left(\partial+\left(a^{\prime}-d^{\prime}\right) \lambda+b\right) h_{0}(\partial+\lambda, 0)=(\partial+(a-d) \lambda+b) h_{0}(\partial, 0) .
$$

Consider $a^{\prime} \neq d^{\prime}, a \neq d$ or $a^{\prime}=d^{\prime}, a=d$ in (4.9). It is straightforward to verify that $h_{0}(\partial, 0)=\sigma \in \mathbb{C}^{*}$. Using this in (4.7) and (4.8), we have

$$
h_{0}(\partial, \lambda)=\sigma \quad \text { and } \quad a^{\prime}=a+1 .
$$

If $p=-1$ in (4.1), one has $c=c^{\prime}$.

Clearly, we need not discuss the case for $a^{\prime} \neq d^{\prime}$ and $a=d$. The final case is $a^{\prime}=d^{\prime}$ and $a \neq d$. Considering the highest degree of $\lambda$ in (4.9) , one can obtain $\operatorname{deg}_{\lambda}\left(h_{0}(\partial+\lambda, 0)\right)=1$. The equation of (4.7) can be written as

$$
h_{0}(\partial, \lambda)+a h_{0}(\partial, 0)-a^{\prime} h_{0}(\partial+\lambda, 0)=(\partial+b) \frac{h_{0}(\partial+\lambda, 0)-h_{0}(\partial, 0)}{\lambda} .
$$

Taking $\lambda \rightarrow 0$ in (4.10), we get $\left(a-a^{\prime}+1\right) h_{0}(\partial, 0)=(\partial+b) \frac{d}{d \partial}\left(h_{0}(\partial, 0)\right)$, which implies

$$
a^{\prime}=a \quad \text { and } \quad h_{0}(\partial, 0)=\sigma(\partial+b)
$$

for $\sigma \in \mathbb{C}^{*}$. Now putting (4.11) in (4.7) or (4.8) gives

$$
h_{0}(\partial, \lambda)=\sigma(\partial+a \lambda+b)
$$

If $p=-1$ in (4.1), we have $c=c^{\prime}=0$.

Case 3. $h_{0}(\partial, 0)=0$ and $\widehat{h}_{0}(\partial, 0) \neq 0$. 
By the similar arguments in Case 2, we show that

$$
a^{\prime}=a-1, b^{\prime}=b, d^{\prime}=d-1, \widehat{h}_{0}(\partial, \lambda)=\sigma \text { and if } p=-1, c^{\prime}=c
$$

or

$$
a^{\prime}=a=d, b^{\prime}=b, d^{\prime}=a-1, \widehat{h}_{0}(\partial, \lambda)=\sigma(\partial+a \lambda+b) \text { and if } p=-1, c^{\prime}=c=0,
$$

where $\sigma \in \mathbb{C}^{*}$. This completes the proof.

Now we determine the irreducibilities of conformal modules $V$ over $\mathcal{S}(p)$ defined in Theorem 4.4 .

Proposition 4.5. Let $V$ be a conformal module over $\mathcal{S}(p)$ defined in Theorem 4.4.

(1) If $V \cong V_{a, b, c, d, a^{\prime}, b^{\prime}, c^{\prime}, d^{\prime}}$, then $V$ is reducible, which has some submodules as $\mathbb{C}[\partial] v_{\overline{0}}$ and $\mathbb{C}[\partial] v_{\overline{1}}$

(2) If $V \cong V_{a, b, c, d, \sigma}$, then $V$ is irreducible if and only if $(a, c, d) \neq(0,0,0)$. The module $V_{0, b, 0,0, \sigma}$ contains a unique non-trivial submodule $\mathbb{C}[\partial](\partial+b) v_{\overline{0}} \oplus \mathbb{C}[\partial] v_{\overline{1}} \cong V_{1, b, \sigma}$.

(3) If $V \cong V_{a, b, \sigma}$, then $V$ is irreducible if and only if $a \neq 0$. The module $V_{0, b, \sigma}$ contains $a$ unique non-trivial submodule $\mathbb{C}[\partial] v_{\overline{0}} \oplus \mathbb{C}[\partial](\partial+b) v_{\overline{1}} \cong V_{0, b, 0,-1, \sigma}$.

(4) If $V \cong \Pi\left(V_{a-1, b, c, d-1, \sigma}\right)$, then $V$ is irreducible if and only if $(a, c, d) \neq(1,0,1)$. The module $\Pi\left(V_{0, b, 0,0, \sigma}\right)$ contains a unique non-trivial submodule $\mathbb{C}[\partial] v_{\overline{0}} \oplus \mathbb{C}[\partial](\partial+b) v_{\overline{1}} \cong$ $\Pi\left(V_{1, b, \sigma}\right)$.

(5) If $V \cong \Pi\left(V_{a, b, \sigma}\right)$, then $V$ is irreducible if and only if $a \neq 0$. The module $\Pi\left(V_{0, b, \sigma}\right)$ contains a unique non-trivial submodule $\mathbb{C}[\partial](\partial+b) v_{\overline{0}} \oplus \mathbb{C}[\partial] v_{\overline{1}} \cong \Pi\left(V_{0, b, 0,-1, \sigma}\right)$.

\subsection{Classification theorems}

The following lemma can be found in [7, 16].

Lemma 4.6. Let $\mathcal{L}$ be a Lie superalgebra with a descending sequence of subspaces $\mathcal{L} \supset \mathcal{L}_{0} \supset$ $\mathcal{L}_{1} \supset \cdots$ and an element $\partial$ satisfying $\left[\partial, \mathcal{L}_{n}\right]=\mathcal{L}_{n-1}$ for $n \geq 1$. Let $V$ be an $\mathcal{L}$-module and let

$$
V_{n}=\left\{v \in V \mid \mathcal{L}_{n} v=0\right\}, n \in \mathbb{Z}_{+} .
$$

Suppose that $V_{n} \neq 0$ for $n \gg 0$ and let $N$ denote the minimal such $n$. Suppose that $N \geq 1$. Then $V=\mathbb{C}[\partial] \otimes_{\mathbb{C}} V_{N}$. Particularly, $V_{N}$ is finite-dimensional if $V$ is a finitely generated $\mathbb{C}[\partial]$-module.

The method in Lemma 6.3 of [26] can be expanded to the following results with a slightly different discussion.

Lemma 4.7. Any finite non-trivial irreducible $\mathcal{S}(p)$-module $V$ must be free of rank 1 or $1+1$. 
Proof. It follows from any torsion module of $\mathbb{C}[\partial]$ is trivial as a module of Lie conformal superalgebra that any finite non-trivial irreducible $\mathcal{S}(p)$-module $V$ must be free as a $\mathbb{C}[\partial]$ module. According to Theorem 4.2, one can see that the $\lambda$-actions of $L_{i}, W_{i}$ and $G_{i}$ on $V$ are trivial for all $i \gg 0$. Assume that $t \in \mathbb{Z}_{+}$is the largest integer such that the $\lambda$-action of $\mathcal{S}(p)_{t}$ on $V$ is non-trivial. Thus $V$ can be regarded as a finite non-trivial irreducible conformal module over $\mathcal{S}(p)_{[t]}$. Denote

$$
\mathfrak{g s \mathfrak { b }}=\left\{\bar{L}_{i, m}, \bar{W}_{j, n}, \bar{G}_{k, l}, \partial \mid 0 \leq i, j, k \leq t, n \in \mathbb{Z}_{+}, m, l \in \mathbb{Z}_{+} \cup\{-1\}\right\} .
$$

Based on Proposition [2.4, the conformal $\mathcal{S}(p)_{[t]}$-module $V$ can be viewed as a module over the associated extended annihilation algebra $\mathfrak{g} \mathfrak{s} \mathfrak{b}=\mathcal{A}\left(\mathcal{S}(p)_{[t]}\right)^{e}$, which satisfies

$$
\bar{L}_{i, m} v=\bar{W}_{j, n} v=\bar{G}_{k, l} v=0
$$

for $0 \leq i, j, k \leq t, m, n, l \gg 0, v \in V$. For later use, we write

$$
\mathfrak{g s}^{\mathfrak{b}}{ }_{z}=\left\{\bar{L}_{i, m}, \bar{W}_{j, n}, \bar{G}_{k, l} \in \mathfrak{g} \mathfrak{g} \mathfrak{b} \mid 0 \leq i, j, k \leq t, m, l \geq z-1, n \geq z\right\}, \forall z \in \mathbb{Z}_{+} .
$$

Then $\mathfrak{g s b}_{0}=\mathcal{A}\left(\mathcal{S}(p)_{[t]}\right)$ and $\mathfrak{g} \mathfrak{s} \mathfrak{b} \supset \mathfrak{g s b}_{0} \supset \mathfrak{g s b}_{1} \supset \ldots$. It follows from the definition of extended annihilation algebra that the element $\partial \in \mathfrak{g} \mathfrak{s} \mathfrak{b}$ satisfies $\left[\partial, \mathfrak{g} \mathfrak{s} \mathfrak{b}_{z}\right]=\mathfrak{g s b}_{z-1}$ for $z \geq 1$. Denote

$$
V_{z}=\left\{v \in V \mid \mathfrak{g s}_{z} v=0\right\}, \forall z \in \mathbb{Z}_{+} .
$$

We observe that $V_{z} \neq \emptyset$ for $z \gg 0$ by (4.12). Assume that $N \in \mathbb{Z}_{+}$is the smallest integer such that $V_{N} \neq \emptyset$.

Firstly, consider $N=0$. Let $0 \neq v \in V_{0}$. Then $\mathcal{U}(\mathfrak{g} \mathfrak{s} \mathfrak{b}) v=\mathbb{C}[\partial] \mathcal{U}\left(\mathfrak{g s}_{\mathfrak{s}} \mathfrak{b}_{0}\right) v=\mathbb{C}[\partial] v$. Therefore, from the irreducibility of $V$, we have $V=\mathbb{C}[\partial] v$. It is clear that $\mathfrak{g s}_{0} \mathfrak{b}_{0}$ acts trivially on $V$. According to Proposition 2.4, we know that $V$ is a trivial conformal $\mathcal{S}(p)$-module, which leads to a contradiction.

Secondly, consider $N \geq 1$. Take $0 \neq v \in V_{N}$. From

$$
\left[\partial-\frac{1}{p} \bar{L}_{0,-1}, L_{i, m}\right]=\left[\partial-\frac{1}{p} \bar{L}_{0,-1}, W_{i, m}\right]=\left[\partial-\frac{1}{p} \bar{L}_{0,-1}, G_{i, m}\right]=0
$$

for $i, m \in \mathbb{Z}_{+}$, one can show that $\partial-\frac{1}{p} \bar{L}_{0,-1}$ is an even central element of $\mathfrak{g} \mathfrak{g} \mathfrak{b}$ by the definition of extended annihilation algebra. So there exists some $\varrho \in \mathbb{C}$ such that $\bar{L}_{0,-1} v=p(\partial+\varrho) v$ by Schur's Lemma of Lie superalgebra. Moreover, according to the following relations

$$
\bar{L}_{i,-1} v=\frac{1}{p}\left[\bar{L}_{i, 0}, \bar{L}_{0,-1}\right] v \text { and } \bar{G}_{i,-1} v=\frac{1}{p}\left[\bar{G}_{i, 0}, \bar{L}_{0,-1}\right] v,
$$

we obtain that the action of $\mathfrak{g} \mathfrak{s} \mathfrak{b}_{0}$ on $v$ is determined by $\mathfrak{g s b}_{1}$ and $\partial$. Obviously, $V_{N}$ is $\mathfrak{g} \mathfrak{s} \mathfrak{b}_{1^{-}}$ invariant. By the irreducibility of $V$ and Lemma 4.6, we obtain that $V=\mathbb{C}[\partial] \otimes_{\mathbb{C}} V_{N}$ and $V_{N}$ is a non-trivial irreducible finite-dimensional $\mathfrak{g s}_{\mathfrak{s}} \mathfrak{b}_{1}$-module. 
If $N=1$, from the definition of $V_{1}$, we know that $V_{1}$ is a trivial $\mathfrak{g} \mathfrak{s} \mathfrak{b}_{1}$-module, which gives a contradiction.

If $N \geq 2$, the module $V_{N}$ can be viewed as a $\mathfrak{g} \mathfrak{s} \mathfrak{b}_{1} / \mathfrak{g} \mathfrak{s} \mathfrak{b}_{N}$-module. Note that $\mathfrak{g s} \mathfrak{b}_{1} / \mathfrak{g} \mathfrak{s} \mathfrak{b}_{N} \cong$ $\mathfrak{p}(t, N-2)$. Based on Lemma 3.6, one can see that $V_{N}$ is 1-dimensional or 1+1-dimensional. Then $V$ is free of rank 1 or $1+1$ as a conformal module over $\mathcal{S}(p)$ by Proposition 2.4. The lemma holds.

Note that the $\mathcal{S}(p)_{\overline{0}}$-module $V_{a, b, c, d}$ defined in Lemma 4.1 can be regarded as an $\mathcal{S}(p)$ module by the trivial action of $\mathcal{S}(p)_{\overline{1}}$. Combining Theorem 4.4, Proposition 4.5 and Lemma 4.7, we have the main result of this paper as follows, which shows that the irreducible modules $V$ defined in Theorem 4.4 exhaust all non-trivial finite irreducible conformal modules over $\mathcal{S}(p)$.

Theorem 4.8. Let $V$ be a non-trivial finite irreducible conformal module over $\mathcal{S}(p)$.

(1) If $p \neq-1$, then

$$
V \cong \begin{cases}V_{a, b, 0, d} & \text { for }(a, d) \neq(0,0), b \in \mathbb{C}, \\ V_{a, b, 0, d, \sigma} & \text { for }(a, d) \neq(0,0), b \in \mathbb{C}, \sigma \in \mathbb{C}^{*}, \\ V_{a, b, \sigma} & \text { for } a, \sigma \in \mathbb{C}^{*}, b \in \mathbb{C}, \\ \Pi\left(V_{a-1, b, 0, d-1, \sigma}\right) & \text { for }(a, d) \neq(1,1), b \in \mathbb{C}, \sigma \in \mathbb{C}^{*}, \\ \Pi\left(V_{a, b, \sigma}\right) & \text { for } a, \sigma \in \mathbb{C}^{*}, b \in \mathbb{C}\end{cases}
$$

(2) If $p=-1$, then

$$
V \cong \begin{cases}V_{a, b, c, d} & \text { for }(a, c, d) \neq(0,0,0), b \in \mathbb{C}, \\ V_{a, b, c, d, \sigma} & \text { for }(a, c, d) \neq(0,0,0), b \in \mathbb{C}, \sigma \in \mathbb{C}^{*}, \\ V_{a, b, \sigma} & \text { for } a, \sigma \in \mathbb{C}^{*}, b \in \mathbb{C}, \\ \Pi\left(V_{a-1, b, c, d-1, \sigma}\right) & \text { for }(a, c, d) \neq(1,0,1), b \in \mathbb{C}, \sigma \in \mathbb{C}^{*}, \\ \Pi\left(V_{a, b, \sigma}\right) & \text { for } a, \sigma \in \mathbb{C}^{*}, b \in \mathbb{C} .\end{cases}
$$

\section{$5 \quad$ Generalized version of $\mathcal{S}(p)$}

The aim of this section is to construct a class of new Lie conformal superalgebras as an extended case of $\mathcal{S}(p)$.

Now, we continue to apply the $\mathfrak{B}(p, 0, p)$-module $V\left(\alpha_{1}, \beta_{1}, \gamma_{1}, p\right)$ in (3.1) and define a super module $V=V_{\overline{0}} \oplus V_{\overline{1}}$, where $V_{\overline{0}}=\bigoplus_{i \in \mathbb{Z}} \mathbb{C}[\partial] v_{\overline{0}}^{i}$ and $V_{\overline{1}}=\oplus_{i \in \mathbb{Z}} \mathbb{C}[\partial] v_{\overline{1}}^{i}$. More precisely, for $\alpha_{1}, \beta_{1}, \gamma_{1}, \widehat{\alpha}_{1}, \widehat{\beta}_{1}, \widehat{\gamma}_{1} \in \mathbb{C}, p \in \mathbb{C}^{*}$, the $\mathbb{C}[\partial]$-module

$$
V\left(\alpha_{1}, \beta_{1}, \gamma_{1}, \widehat{\alpha}_{1}, \widehat{\beta}_{1}, \widehat{\gamma}_{1}, p\right)=\oplus_{i \in \mathbb{Z}} \mathbb{C}[\partial] v_{\overline{0}}^{i} \bigoplus \oplus_{i \in \mathbb{Z}} \mathbb{C}[\partial] v_{\overline{1}}^{i}
$$


is a $\mathbb{Z}$-graded free intermediate series module of rank two over $\mathfrak{B}(p, 0, p)$ with $\lambda$-actions as follows:

$$
\begin{aligned}
& L_{i \lambda} v_{\overline{0}}^{j}=\left((i+p)\left(\partial+\beta_{1}\right)+\left(i+j+\alpha_{1}\right) \lambda\right) v_{\overline{0}}^{i+j}, W_{i \lambda} v_{\overline{0}}^{j}=\gamma_{1} v_{\overline{0}}^{i+j} \\
& L_{i \lambda} v_{\overline{1}}^{j}=\left((i+p)\left(\partial+\widehat{\beta}_{1}\right)+\left(i+j+\widehat{\alpha}_{1}\right) \lambda\right) v_{\overline{1}}^{i+j}, W_{i \lambda} v_{\overline{1}}^{j}=\widehat{\gamma}_{1} v_{\overline{1}}^{i+j}
\end{aligned}
$$

We consider a $\mathbb{Z}_{2}$-graded $\mathbb{C}[\partial]$-module

$$
\mathcal{G S}\left(\alpha_{1}, \beta_{1}, \gamma_{1}, \widehat{\alpha}_{1}, \widehat{\beta}_{1}, \widehat{\gamma}_{1},\left\{\phi_{i, j}, \varphi_{i, j}\right\}\right)=\mathcal{G S}_{\overline{0}} \oplus \mathcal{G S}_{\overline{1}}
$$

with $\mathcal{G S}_{\overline{0}}=\oplus_{i \in \mathbb{Z}_{+}} \mathbb{C}[\partial] L_{i} \bigoplus \oplus_{i \in \mathbb{Z}_{+}} \mathbb{C}[\partial] W_{i}, \mathcal{G} \mathcal{S}_{\overline{1}}=\oplus_{i \in \mathbb{Z}_{+}} \mathbb{C}[\partial] G_{i} \bigoplus \oplus_{i \in \mathbb{Z}_{+}} \mathbb{C}[\partial] H_{i}$ and satisfying

$$
\begin{aligned}
& {\left[L_{i \lambda} L_{j}\right]=((i+p) \partial+(i+j+2 p) \lambda) L_{i+j},} \\
& {\left[L_{i \lambda} W_{j}\right]=((i+p)(\partial+\beta)+(i+j+\alpha) \lambda) W_{i+j}} \\
& {\left[L_{i \lambda} G_{j}\right]=\left((i+p)\left(\partial+\beta_{1}\right)+\left(i+j+\alpha_{1}\right) \lambda\right) G_{i+j},} \\
& {\left[W_{i \lambda} G_{j}\right]=\gamma_{1} G_{i+j},} \\
& {\left[L_{i \lambda} H_{j}\right]=\left((i+p)\left(\partial+\widehat{\beta}_{1}\right)+\left(i+j+\widehat{\alpha}_{1}\right) \lambda\right) H_{i+j},} \\
& {\left[W_{i \lambda} H_{j}\right]=\widehat{\gamma_{1}} H_{i+j},} \\
& {\left[G_{i \lambda} H_{j}\right]=\phi_{i, j}(\partial, \lambda) W_{i+j}+\varphi_{i, j}(\partial, \lambda) L_{i+j},} \\
& {\left[W_{i \lambda} W_{j}\right]=\left[G_{i \lambda} G_{j}\right]=\left[H_{i \lambda} H_{j}\right]=0,}
\end{aligned}
$$

where $\phi_{i, j}(\partial, \lambda), \varphi_{i, j}(\partial, \lambda) \in \mathbb{C}[\partial, \lambda]$ for $i, j \in \mathbb{Z}_{+}$.

Lemma 5.1. Let $p \in \mathbb{C}^{*}, \alpha_{1}=2 p, \widehat{\alpha}_{1}=p, \beta_{1}=\widehat{\beta}_{1}=0, \gamma_{1}=1$ and $\widehat{\gamma}_{1}=-1$. Then $\mathbb{Z}_{2}$-graded $\mathbb{C}[\partial]$-module $\mathcal{G S}\left(\alpha_{1}, \beta_{1}, \gamma_{1}, \widehat{\alpha}_{1}, \widehat{\beta}_{1}, \widehat{\gamma}_{1},\left\{\phi_{i, j}, \varphi_{i, j}\right\}\right)$ becomes a Lie conformal superalgebra if and only if $\phi_{i, j}(\partial, \lambda)=\Delta$ and $\varphi_{i, j}(\partial, \lambda)=\Delta((i+p) \partial+(i+j+p) \lambda)$ for $i, j \in \mathbb{Z}_{+}, \Delta \in \mathbb{C}$.

Proof. It follows from Definition 2.1 that the sufficiency is clear.

We only need to prove the necessity. Assume that $\mathcal{G S}\left(\alpha_{1}, \beta_{1}, \gamma_{1}, \widehat{\alpha}_{1}, \widehat{\beta}_{1}, \widehat{\gamma}_{1},\left\{\phi_{i, j}, \varphi_{i, j}\right\}\right)$ is a Lie conformal superalgebra. For any $i, j \in \mathbb{Z}_{+}$, using the Jacobi identity for triple $\left(W_{0}, G_{i}, H_{j}\right)$, we have

$$
\begin{aligned}
& \varphi_{i, j}(\partial, \lambda+\mu)=\varphi_{i, j}(\partial, \mu), \\
& (i+j+p) \lambda \varphi_{i, j}(\partial+\lambda, \mu)=\phi_{i, j}(\partial, \lambda+\mu)-\phi_{i, j}(\partial, \mu) .
\end{aligned}
$$

From (5.1), one has

$$
\varphi_{i, j}(\partial, \lambda)=\varphi_{i, j}(\partial, 0)
$$


for $i, j \in \mathbb{Z}_{+}$. For any $i, j \in \mathbb{Z}_{+}$, by the Jacobi identity for triple $\left(L_{0}, G_{i}, H_{j}\right)$, we check that

$$
\begin{aligned}
& (p \partial+(i+j+p) \lambda) \phi_{i, j}(\partial+\lambda, \mu) \\
= & ((i+p) \lambda-p \mu) \phi_{i, j}(\partial, \lambda+\mu)+(p(\partial+\mu)+(j+p) \lambda) \phi_{i, j}(\partial, \mu), \\
& (p \partial+(i+j+2 p) \lambda) \varphi_{i, j}(\partial+\lambda, \mu) \\
= & ((i+p) \lambda-p \mu) \varphi_{i, j}(\partial, \lambda+\mu)+(p(\partial+\mu)+(j+p) \lambda) \varphi_{i, j}(\partial, \mu) .
\end{aligned}
$$

Setting $\mu=0$ in (5.5) and using (15.3), we conclude that $\varphi_{i, j}(\partial, \lambda)=\Delta \in \mathbb{C}$ for any $i, j \in \mathbb{Z}_{+}$. We take $\mu=0$ in (5.2), one can write

$$
\phi_{i, j}(\partial, \lambda)=\phi_{i, j}(\partial, 0)+\Delta(i+j+p) \lambda
$$

By choosing $\mu=0$ in (5.4), it is easy to show that

$$
\begin{aligned}
& p \partial \frac{\left(\phi_{i, j}(\partial+\lambda, 0)-\phi_{i, j}(\partial, 0)\right)}{\lambda} \\
= & (i+p) \phi_{i, j}(\partial, \lambda)+(j+p) \phi_{i, j}(\partial, 0)-(i+j+p) \phi_{i, j}(\partial+\lambda, 0) .
\end{aligned}
$$

Taking $\lambda \rightarrow 0$, we have $\partial \frac{d}{d \partial} \phi_{i, j}(\partial, 0)=\phi_{i, j}(\partial, 0)$, which has a nonzero solution $\phi_{i, j}(\partial, 0)=$ $\Delta_{0}(i+p) \partial$ for $\Delta_{0} \in \mathbb{C}$. Then (5.6) can be written as

$$
\phi_{i, j}(\partial, \lambda)=\Delta_{0}(i+p) \partial+\Delta(i+j+p) \lambda
$$

Inserting (5.8) into (5.7), we immediately obtain $\Delta=\Delta_{0}$, which yields $\phi_{i, j}(\partial, \lambda)=\Delta((i+$ $p) \partial+(i+j+p) \lambda)$ for $i, j \in \mathbb{Z}_{+}, \Delta \in \mathbb{C}$. We complete the proof.

Remark 5.2. Up to isomorphism, we may assume that $\Delta=2$ in Lemma 5.1 for $\Delta \neq 0$. Then we can define a class of Lie conformal superalgebras $\mathcal{G S}(p)=\mathcal{G S}_{\overline{0}} \oplus \mathcal{G S}_{\overline{1}}$ with

$$
\mathcal{G S}_{\overline{0}}=\oplus_{i \in \mathbb{Z}_{+}} \mathbb{C}[\partial] L_{i} \bigoplus \oplus_{i \in \mathbb{Z}_{+}} \mathbb{C}[\partial] W_{i}, \mathcal{G S}_{\overline{1}}=\oplus_{i \in \mathbb{Z}_{+}} \mathbb{C}[\partial] G_{i} \bigoplus \oplus_{i \in \mathbb{Z}_{+}} \mathbb{C}[\partial] H_{i}
$$

and the following non-trivial $\lambda$-brackets

$$
\begin{aligned}
& {\left[L_{i \lambda} L_{j}\right]=((i+p) \partial+(i+j+2 p) \lambda) L_{i+j},} \\
& {\left[L_{i \lambda} W_{j}\right]=((i+p) \partial+(i+j+p) \lambda) W_{i+j},} \\
& {\left[L_{i \lambda} G_{j}\right]=((i+p) \partial+(i+j+2 p) \lambda) G_{i+j},} \\
& {\left[L_{i \lambda} H_{j}\right]=((i+p) \partial+(i+j+p) \lambda) H_{i+j},} \\
& {\left[W_{i \lambda} G_{j}\right]=G_{i+j},\left[W_{i \lambda} H_{j}\right]=-H_{i+j},} \\
& {\left[G_{i \lambda} H_{j}\right]=2 L_{i+j}+2((i+p) \partial+(i+j+p) \lambda) W_{i+j} .}
\end{aligned}
$$


Denote the subalgebra $\mathbb{C}[\partial] L_{0} \oplus \mathbb{C}[\partial] W_{0} \oplus \mathbb{C}[\partial] G_{0} \oplus \mathbb{C}[\partial] H_{0}$ of $\mathcal{G S}(p)$ by $\mathcal{S} \mathcal{N}$. Define the following $\mathbb{C}[\partial]$-module homomorphism from $\mathcal{S N}$ to the Lie conformal algebra of $N=2$ superconformal algebra (see [9]):

$$
\frac{1}{p} L_{0}+\frac{1}{2} \partial W_{0} \rightarrow L, W_{0} \rightarrow J, G_{0} \rightarrow G_{+}, \frac{1}{p} H_{0} \rightarrow G_{-} .
$$

It is easy to check that the subalgebra $\mathcal{S N}$ is isomorphic to the Lie conformal algebra of $N=2$ superconformal algebra.

A class of infinite-dimensional Lie superalgebras related to Block type Lie algebra are presented in the rest of this section, which have a subalgebra called topological $N=2$ superconformal algebra.

Lemma 5.3. The annihilation superalgebra of $\mathcal{G S}(p)$ is given by

$$
\mathcal{A}(\mathcal{G S}(p))=\left\{L_{i, m}, W_{j, n}, G_{k, l}, H_{p, q} \mid i, j, k, n \in \mathbb{Z}_{+}, m, l \in \mathbb{Z}_{+} \cup\{-1\}\right\}
$$

with non-vanishing relations:

$$
\begin{aligned}
& {\left[L_{i, m}, L_{j, n}\right]=((m+1)(j+p)-(n+1)(i+p)) L_{i+j, m+n},} \\
& {\left[L_{i, m}, W_{j, n}\right]=((m+1) j-n(i+p)) W_{i+j, m+n},} \\
& {\left[L_{i, m}, G_{j, n}\right]=((m+1)(j+p)-(n+1)(i+p)) G_{i+j, m+n},} \\
& {\left[L_{i, m}, H_{j, n}\right]=((m+1) j-n(i+p)) H_{i+j, m+n},} \\
& {\left[G_{i, m}, H_{j, n}\right]=2 L_{i+j, m+n}+2((m+1) j-n(i+p)) W_{i+j, m+n},} \\
& {\left[W_{i, m}, G_{j, n}\right]=G_{i+j, m+n},\left[W_{i, m}, H_{j, n}\right]=-H_{i+j, m+n},}
\end{aligned}
$$

where $p \in \mathbb{C}^{*}$.

Proof. Since the proof is similar to Lemma 3.3, we omit the details.

Remark 5.4. We observe that the topological $N=2$ superconformal algebra is isomorphic to the Lie superalgebra generated by $\left\{L_{0, m}, W_{0, n}, G_{0, l}, H_{0, q} \mid m, n, l, q \in \mathbb{Z}\right\}$ (see [13]).

\section{Applications}

From the definition of (3.4), we know that $\mathcal{S}(p)_{[n]}$ has a $\mathbb{C}[\partial]$-basis $\left\{\bar{L}_{i}, \bar{W}_{i}, \bar{G}_{i} \mid 0 \leq i \leq n\right\}$ with the following $\lambda$-brackets:

$$
\begin{aligned}
& {\left[\bar{L}_{i \lambda} \bar{L}_{j}\right]=((i+p) \partial+(i+j+2 p) \lambda) \bar{L}_{i+j},} \\
& {\left[\bar{L}_{i \lambda} \bar{W}_{j}\right]=((i+p) \partial+(i+j+p) \lambda) \bar{W}_{i+j},} \\
& {\left[\bar{L}_{i \lambda} \bar{G}_{j}\right]=((i+p) \partial+(i+j+2 p) \lambda) \bar{G}_{i+j},} \\
& {\left[\bar{W}_{i \lambda} \bar{G}_{j}\right]=\bar{G}_{i+j}, \quad\left[\bar{W}_{i \lambda} \bar{W}_{j}\right]=\left[\bar{G}_{i \lambda} \bar{G}_{j}\right]=0}
\end{aligned}
$$


for $i, j \in \mathbb{Z}_{+}, p \in \mathbb{C}^{*}(i+j>n$ the above relations are trivial $)$. It follows from that $\mathcal{S}(p)_{[0]} \cong \mathfrak{s h}, \mathfrak{s}(n)=\mathcal{S}(-n)_{[n]}$ for $n \geq 1$ (also see (3.4), (3.5)). Now we define $\mathbb{C}[\partial]$-modules $\bar{V}_{a, b, 0, d}, \bar{V}_{a, b, 0, d, \sigma}$ and $\bar{V}_{a, b, \sigma}$ over $\mathfrak{s}(n)$ for $n>1$ as follows.

(1) $\bar{V}_{a, b, 0, d}=\mathbb{C}[\partial] v$ with

$$
\left\{\begin{array}{l}
\bar{L}_{0 \lambda} v=-n(\partial+a \lambda+b) v \\
\bar{W}_{0 \lambda} v=d v \\
\bar{G}_{i \lambda} v=0,0 \leq i \leq n \\
\bar{W}_{j \lambda} v=0,1 \leq j \leq n \\
\bar{L}_{k \lambda} v=0,1 \leq k \leq n
\end{array}\right.
$$

where $a, b, d \in \mathbb{C}$;

(2) $\bar{V}_{a, b, 0, d, \sigma}=\mathbb{C}[\partial] v_{\overline{0}} \oplus \mathbb{C}[\partial] v_{\overline{1}}$ with

$$
\left\{\begin{array} { l } 
{ \overline { L } _ { 0 \lambda } v _ { \overline { 0 } } = - n ( \partial + a \lambda + b ) v _ { \overline { 0 } } , } \\
{ \overline { W } _ { 0 \lambda } v _ { \overline { 0 } } = d v _ { \overline { 0 } } , } \\
{ \overline { G } _ { 0 \lambda } v _ { \overline { 0 } } = \sigma v _ { \overline { 1 } } , } \\
{ \overline { G } _ { i \lambda } v _ { \overline { 0 } } = 0 , 1 \leq i \leq n , } \\
{ \overline { W } _ { j \lambda } v _ { \overline { 0 } } = 0 , 1 \leq j \leq n , } \\
{ \overline { L } _ { k \lambda } v _ { \overline { 0 } } = 0 , 1 \leq k \leq n , }
\end{array} \quad \text { nd } \left\{\begin{array}{l}
\bar{L}_{0 \lambda} v_{\overline{1}}=-n(\partial+(a+1) \lambda+b) v_{\overline{1}}, \\
\bar{W}_{0 \lambda} v_{\overline{1}}=(d+1) v_{\overline{1}}, \\
\bar{G}_{i \lambda} v_{\overline{1}}=0,0 \leq i \leq n, \\
\bar{W}_{j \lambda} v_{\overline{1}}=0,1 \leq j \leq n, \\
\bar{L}_{k \lambda} v_{\overline{1}}=0,1 \leq k \leq n,
\end{array}\right.\right.
$$

where $a, b, d \in \mathbb{C}, \sigma \in \mathbb{C}^{*}$

(3) $\bar{V}_{a, b, \sigma}=\mathbb{C}[\partial] v_{\overline{0}} \oplus \mathbb{C}[\partial] v_{\overline{1}}$ with

$$
\left\{\begin{array}{l}
\bar{L}_{0 \lambda} v_{\overline{0}}=-n(\partial+a \lambda+b) v_{\overline{0}}, \\
\bar{W}_{0 \lambda} v_{\overline{0}}=(a-1) v_{\overline{0}}, \\
\bar{G}_{0 \lambda} v_{\overline{0}}=\sigma(\partial+a \lambda+b) v_{\overline{1}}, \quad \text { and } \quad\left\{\begin{array}{l}
\bar{L}_{0 \lambda} v_{\overline{1}}=-n(\partial+a \lambda+b) v_{\overline{1}}, \\
\bar{W}_{0 \lambda} v_{\overline{1}}=a v_{\overline{1}}, \\
\bar{G}_{i \lambda} v_{\overline{0}}=0,1 \leq i \leq n, \\
\bar{W}_{j \lambda} v_{\overline{1}}=0,1 \leq j \leq n, 0 \leq i \leq n, \\
\bar{W}_{j \lambda} v_{\overline{1}}=0,1 \leq j \leq n, \\
\bar{L}_{k \lambda} v_{\overline{0}}=0,1 \leq k \leq n,
\end{array} \quad \text { vis } 1 \leq k \leq n,\right.
\end{array}\right.
$$

where $a, b \in \mathbb{C}, \sigma \in \mathbb{C}^{*}$.

The $\mathbb{C}[\partial]$-modules $\overline{\bar{V}}_{a, b, c, d}, \overline{\bar{V}}_{a, b, c, d, \sigma}$ and $\overline{\bar{V}}_{a, b, \sigma}$ over $\mathfrak{s}(1)$ are given. 
(1) $\overline{\bar{V}}_{a, b, c, d}=\mathbb{C}[\partial] v$ with

$$
\left\{\begin{array}{l}
L_{0 \lambda} v=-(\partial+a \lambda+b) v \\
L_{1 \lambda} v=c v \\
W_{0 \lambda} v=d v \\
G_{i \lambda} v=0,0 \leq i \leq 1 \\
W_{1 \lambda} v=0
\end{array}\right.
$$

where $a, b, c, d \in \mathbb{C}$;

(2) $\overline{\bar{V}}_{a, b, c, d, \sigma}=\mathbb{C}[\partial] v_{\overline{0}} \oplus \mathbb{C}[\partial] v_{\overline{1}}$ with

$$
\left\{\begin{array} { l } 
{ L _ { 0 \lambda } v _ { \overline { 0 } } = - ( \partial + a \lambda + b ) v _ { \overline { 0 } } , } \\
{ L _ { 1 \lambda } v _ { \overline { 0 } } = c v _ { \overline { 0 } } , } \\
{ W _ { 0 \lambda } v _ { \overline { 0 } } = d v _ { \overline { 0 } } , } \\
{ G _ { 0 \lambda } v _ { \overline { 0 } } = \sigma v _ { \overline { 1 } } , } \\
{ G _ { 1 \lambda } v _ { \overline { 0 } } = W _ { 1 \lambda } v _ { \overline { 0 } } = 0 , }
\end{array} \quad \text { and } \left\{\begin{array}{l}
L_{0 \lambda} v_{\overline{1}}=-(\partial+(a+1) \lambda+b) v_{\overline{1}}, \\
L_{1 \lambda} v_{\overline{1}}=c v_{\overline{1}}, \\
W_{0 \lambda} v_{\overline{1}}=(d+1) v_{\overline{1}}, \\
G_{i \lambda} v_{\overline{1}}=0,0 \leq i \leq 1, \\
W_{1 \lambda} v_{\overline{1}}=0,
\end{array}\right.\right.
$$

where $a, b, c, d \in \mathbb{C}, \sigma \in \mathbb{C}^{*}$;

(3) $\overline{\bar{V}}_{a, b, \sigma}=\mathbb{C}[\partial] v_{\overline{0}} \oplus \mathbb{C}[\partial] v_{\overline{1}}$ with

$$
\left\{\begin{array} { l } 
{ L _ { 0 \lambda } v _ { \overline { 0 } } = - ( \partial + a \lambda + b ) v _ { \overline { 0 } } , } \\
{ W _ { 0 \lambda } v _ { \overline { 0 } } = ( a - 1 ) v _ { \overline { 0 } } , } \\
{ G _ { 0 \lambda } v _ { \overline { 0 } } = \sigma ( \partial + a \lambda + b ) v _ { \overline { 1 } } , } \\
{ G _ { 1 \lambda } v _ { \overline { 0 } } = W _ { 1 \lambda } v _ { \overline { 0 } } = L _ { 1 \lambda } v _ { \overline { 0 } } = 0 , }
\end{array} \quad \text { and } \quad \left\{\begin{array}{l}
L_{0 \lambda} v_{\overline{1}}=-(\partial+a \lambda+b) v_{\overline{1}}, \\
W_{0 \lambda} v_{\overline{1}}=a v_{\overline{1}}, \\
G_{i \lambda} v_{\overline{1}}=0,0 \leq i \leq 1, \\
W_{1 \lambda} v_{\overline{1}}=L_{1 \lambda} v_{\overline{1}}=0,
\end{array}\right.\right.
$$

where $a, b \in \mathbb{C}, \sigma \in \mathbb{C}^{*}$.

The following $\mathbb{C}[\partial]$-modules $\overline{\bar{V}}_{a, b, 0, d}, \overline{\overline{\bar{V}}}_{a, b, 0, d, \sigma}$ and $\overline{\overline{\bar{V}}}_{a, b, \sigma}$ over $\mathfrak{s h}$ are presented.

(1) $\stackrel{\overline{\bar{V}}}{a, b, 0, d}=\mathbb{C}[\partial] v$ with

$$
\left\{\begin{array}{l}
\left(\frac{1}{p} L_{0}\right)_{\lambda} v=(\partial+a \lambda+b) v \\
W_{0 \lambda} v=d v \\
G_{0 \lambda} v=0
\end{array}\right.
$$

where $a, b, d \in \mathbb{C}$; 
(2) $\stackrel{\overline{\bar{V}}}{a, b, 0, d, \sigma}=\mathbb{C}[\partial] v_{\overline{0}} \oplus \mathbb{C}[\partial] v_{\overline{1}}$ with

$$
\left\{\begin{array} { l } 
{ ( \frac { 1 } { p } L _ { 0 } ) _ { \lambda } v _ { \overline { 0 } } = ( \partial + a \lambda + b ) v _ { \overline { 0 } } , } \\
{ W _ { 0 \lambda } v _ { \overline { 0 } } = d v _ { \overline { 0 } } , } \\
{ G _ { 0 \lambda } v _ { \overline { 0 } } = \sigma v _ { \overline { 1 } } , }
\end{array} \quad \text { and } \left\{\begin{array}{l}
\left(\frac{1}{p} L_{0}\right)_{\lambda} v_{\overline{1}}=(\partial+(a+1) \lambda+b) v_{\overline{1}}, \\
W_{0 \lambda} v_{\overline{1}}=(d+1) v_{\overline{1}}, \\
G_{0 \lambda} v_{\overline{1}}=0,
\end{array}\right.\right.
$$

where $a, b, d \in \mathbb{C}, \sigma \in \mathbb{C}^{*}$;

(3) $\overline{\bar{V}}_{a, b, \sigma}=\mathbb{C}[\partial] v_{\overline{0}} \oplus \mathbb{C}[\partial] v_{\overline{1}}$ with

$$
\left\{\begin{array} { l } 
{ ( \frac { 1 } { p } L _ { 0 } ) _ { \lambda } v _ { \overline { 0 } } = ( \partial + a \lambda + b ) v _ { \overline { 0 } } , } \\
{ W _ { 0 \lambda } v _ { \overline { 0 } } = ( a - 1 ) v _ { \overline { 0 } } , } \\
{ G _ { 0 \lambda } v _ { \overline { 0 } } = \sigma ( \partial + a \lambda + b ) v _ { \overline { 1 } } , }
\end{array} \quad \text { and } \quad \left\{\begin{array}{l}
\left(\frac{1}{p} L_{0}\right)_{\lambda} v_{\overline{1}}=(\partial+a \lambda+b) v_{\overline{1}}, \\
W_{0 \lambda} v_{\overline{1}}=a v_{\overline{1}}, \\
G_{0 \lambda} v_{\overline{1}}=0,
\end{array}\right.\right.
$$

where $a, b \in \mathbb{C}, \sigma \in \mathbb{C}^{*}$.

Based on Theorem 4.4, the classification of non-trivial conformal modules of rank $1+1$ over $\mathfrak{s h}$ and $\mathfrak{s}(n)$ for $n \geq 1$ can be presented. Moreover, the same irreducibility assertions as those modules of $\mathfrak{s h}$ and $\mathfrak{s}(n)$ for $n \geq 1$ in Proposition 4.5 are obtained. We see that the irreducible modules of these modules exhaust all non-trivial finite irreducible conformal modules respectively over $\mathfrak{s h}$ and $\mathfrak{s}(n)$ for $n \geq 1$.

Corollary 6.1. Assume that $\bar{V}$ is a finite non-trivial irreducible conformal module over $\mathfrak{s h}$ or $\mathfrak{s}(n)$ for $n \geq 1$.

(i) If $\bar{V}$ is an $\mathfrak{s}(n)$-module for $n>1$, then

$$
\bar{V} \cong \begin{cases}\bar{V}_{a, b, 0, d} & \text { for }(a, d) \neq(0,0), b \in \mathbb{C}, \\ \bar{V}_{a, b, 0, d, \sigma} & \text { for }(a, d) \neq(0,0), b \in \mathbb{C}, \sigma \in \mathbb{C}^{*}, \\ \bar{V}_{a, b, \sigma} & \text { for } a, \sigma \in \mathbb{C}^{*}, b \in \mathbb{C}, \\ \Pi\left(\bar{V}_{a-1, b, 0, d-1, \sigma}\right) & \text { for }(a, d) \neq(1,1), b \in \mathbb{C}, \sigma \in \mathbb{C}^{*}, \\ \Pi\left(\bar{V}_{a, b, \sigma}\right) & \text { for } a, \sigma \in \mathbb{C}^{*}, b \in \mathbb{C} ;\end{cases}
$$

(ii) If $\bar{V}$ is an $\mathfrak{s}(1)$-module, then

$$
\bar{V} \cong \begin{cases}\overline{\bar{V}}_{a, b, c, d} & \text { for }(a, c, d) \neq(0,0,0), b \in \mathbb{C}, \\ \overline{\bar{V}}_{a, b, c, d, \sigma} & \text { for }(a, c, d) \neq(0,0,0), \sigma \in \mathbb{C}^{*}, b \in \mathbb{C}, \\ \overline{\bar{V}}_{a, b, \sigma} & \text { for } a, \sigma \in \mathbb{C}^{*}, b \in \mathbb{C}, \\ \Pi\left(\overline{\bar{V}}_{a-1, b, c, d-1, \sigma}\right) & \text { for }(a, c, d) \neq(1,0,1), \sigma \in \mathbb{C}^{*}, b \in \mathbb{C}, \\ \Pi\left(\overline{\bar{V}}_{a, b, \sigma}\right) & \text { for } a, \sigma \in \mathbb{C}^{*}, b \in \mathbb{C} ;\end{cases}
$$


(iii) If $\bar{V}$ is an $\mathfrak{s h}$-module, then

$$
\bar{V} \cong \begin{cases}\overline{\bar{V}}_{a, b, 0, d} & \text { for }(a, d) \neq(0,0), b \in \mathbb{C}, \\ \overline{\bar{V}}_{a, b, 0, d, \sigma} & \text { for }(a, d) \neq(0,0), \sigma \in \mathbb{C}^{*}, b \in \mathbb{C}, \\ \overline{\bar{V}}_{a, b, \sigma} & \text { for } a, \sigma \in \mathbb{C}^{*}, b \in \mathbb{C}, \\ \Pi\left(\overline{\bar{V}}_{a-1, b, 0, d-1, \sigma}\right) & \text { for }(a, d) \neq(1,1), \sigma \in \mathbb{C}^{*}, b \in \mathbb{C}, \\ \Pi\left(\overline{\bar{V}}_{a, b, \sigma}\right) & \text { for } a, \sigma \in \mathbb{C}^{*}, b \in \mathbb{C} .\end{cases}
$$

Remark 6.2. By (3.2), we also obtain the classification of all non-trivial finite irreducible conformal modules over a subalgebra of Lie conformal algebra of $N=2$ superconformal algebra.

\section{Acknowledgements}

This work was supported by the National Natural Science Foundation of China (No.11801369, 11871421, 11971350), the Zhejiang Provincial Natural Science Foundation of China (No. LY20A010022) and the Scientific Research Foundation of Hangzhou Normal University (No. 2019QDL012). We also thank the referee for nice suggestions to make the paper more readable.

\section{Data Availability}

Data sharing no applicable to this article.

\section{References}

[1] B. Bakalov, V. G. Kac, A. A. Voronov, Cohomology of conformal algebras, Comm. Math. Phys. 200, 561-598 (1999).

[2] C. Boyallian, V. G. Kac, J. I. Liberati, Classification of finite irreducible modules over the Lie conformal superalgebra $C K_{6}$, Comm. Math. Phys. 317, 503-546 (2013).

[3] C. Boyallian, V. G. Kac, J. I. Liberati, Irreducible modules over finite simple Lie conformal superalgebras of type K, J. Math. Phys. 51, 063507 (2010).

[4] C. Boyallian, V. G. Kac, J. I. Liberati, A. Rudakov, Representations of simple finite Lie conformal superalgebras of type $W$ and S, J. Math. Phys. 47, 043513 (2006).

[5] H. Chen, J. Han, Y. Su, Y. Xu, Loop Schrödinger-Virasoro Lie conformal algebra, Internat. J. Math. 27, 1650057 (2016).

[6] H. Chen, Y. Hong, Y. Su, Finite irreducible conformal modules over the extended Block type Lie conformal algebra $\mathfrak{B}(\alpha, \beta, p)$, arXiv:1908.05827. 
[7] S. Cheng, V. G. Kac, Conformal modules, Asian J. Math. 1, 181-193 (1997), Asian J. Math. 2, 153-156 (1998) (Erratum).

[8] S. Cheng, V. G. Kac, M. Wakimoto, Extensions of conformal modules, in: Topological Field Theory, Primitive Forms and Related Topics(Kyoto), in: Progress in Math. 160, Birkhäuser, Boston, 33-57; q-alg/9709019 (1998).

[9] S. Cheng, N. Lam, Finite conformal modules over $N=2,3,4$ superconformal algebras, $J$. Math. Phys. 42, 906-933 (2001).

[10] X. Dai, J. Han, Loop Virasoro Lie conformal superalgebra, Linear Multilinear Algebra 66, 131-146 (2018).

[11] A. D'Andrea, V. G. Kac, Structure theory of finite conformal algebras, Sel. Math. 4, 377-418 (1998).

[12] D. Fattori, V. G. Kac, Classification of finite simple Lie conformal superalgebras, J. Algebra 258, 23-59 (2002).

[13] R. Dijkgraaf, E. Verlinde, H. Verlinde, Topological strings in $d<1$, Nucl. Phys. B 352, 59-86 (1991).

[14] G. Fan, Y. Su, H. Wu, Loop Heisenberg-Virasoro Lie conformal algebra, J. Math. Phys. 55, 123508 (2014).

[15] Y. Hong, Z. Wu, Simplicity of quadratic Lie conformal algebras, Comm. Algebra 45, 141-150 (2017).

[16] V. G. Kac, Vertex algebras for beginners, University Lecture Series Vol. 10, American Mathematical Society, 1996.

[17] V. G. Kac, Formal distribution algebras and conformal algebras, XIIth International Congress of Mathematical Physics (ICMP'97) (Brisbane), 80-97, Int. Press, Cambridge, MA, 1999.

[18] V. G. Kac, The idea of locality, in Physical Applications and Mathematical Aspects of Geometry, Groups and Algebras, World Scientific, Singapore, 16-32 (1997).

[19] L. Luo, Y. Hong, Z. Wu, Finite irreducible modules of Lie conformal algebras $\mathcal{W}(a, b)$ and some Schrödinger-Virasoro type Lie conformal algebras, Int. J. Math. 30, 1950026 (2019).

[20] M. Mansour, On the quantum super Virasoro algebra, Czech. J. Phys. 51, 883-888 (2001).

[21] C. Martinez, E. Zelmanov, Irreducible representations of the exceptional Cheng-Kac superalgebra Trans. Amer. Math. Soc. 366, 5853-5876 (2014).

[22] Y. Su, C. Xia, L. Yuan, Classification of finite irreducible conformal modules over a class of Lie conformal algebras of Block type, J. Algebra 499, 321-336 (2019).

[23] Y. Su, X. Yue, Filtered Lie conformal algebras whose associated graded algebras are isomorphic to that of general conformal algebra $g c_{1}$, J. Algebra 340, 182-198 (2011).

[24] H. Wu, Q. Chen, X. Yue, Loop Virasoro Lie conformal algbera, J. Math. Phys. 55, 011706 (2014). 
[25] H. Wu, L. Yuan, Classification of finite irreducible conformal modules over some Lie conformal algebras related to the Virasoro conformal algebra, J. Math. Phys. 58, 041701 (2017).

[26] C. Xia, Classification of finite irreducible conformal modules over Lie conformal superalgebras of Block type, J. Algebra 531, 141-164 (2019).

\section{Haibo Chen}

School of Statistics and Mathematics, Shanghai Lixin University of Accounting and Finance, Shanghai 201209, China

rebel1025@126.com

Yanyong Hong

Department of Mathematics, Hangzhou Normal University, Hangzhou 311121, China

hongyanyong2008@yahoo.com

Yucai Su

School of Mathematical Sciences, Tongji University, Shanghai 200092, China

ycsu@tongji.edu.cn 